ESAIM: M2AN 50 (2016) 289-309

DOI: $10.1051 / \mathrm{m} 2 \mathrm{an} / 2015044$
ESAIM: Mathematical Modelling and Numerical Analysis

www.esaim-m2an.org

\title{
ROBUST ARBITRARY ORDER MIXED FINITE ELEMENT METHODS FOR THE INCOMPRESSIBLE STOKES EQUATIONS WITH PRESSURE INDEPENDENT VELOCITY ERRORS
}

\author{
Alexander Linke ${ }^{1}$, Gunar Matthies $^{2}$ and Lutz Tobiska ${ }^{3}$
}

\begin{abstract}
Standard mixed finite element methods for the incompressible Navier-Stokes equations that relax the divergence constraint are not robust against large irrotational forces in the momentum balance and the velocity error depends on the continuous pressure. This robustness issue can be completely cured by using divergence-free mixed finite elements which deliver pressure-independent velocity error estimates. However, the construction of $\mathrm{H}^{1}$-conforming, divergence-free mixed finite element methods is rather difficult. Instead, we present a novel approach for the construction of arbitrary order mixed finite element methods which deliver pressure-independent velocity errors. The approach does not change the trial functions but replaces discretely divergence-free test functions in some operators of the weak formulation by divergence-free ones. This modification is applied to inf-sup stable conforming and nonconforming mixed finite element methods of arbitrary order in two and three dimensions. Optimal estimates for the incompressible Stokes equations are proved for the $\mathrm{H}^{1}$ and $\mathrm{L}^{2}$ errors of the velocity and the $\mathrm{L}^{2}$ error of the pressure. Moreover, both velocity errors are pressure-independent, demonstrating the improved robustness. Several numerical examples illustrate the results.
\end{abstract}

Mathematics Subject Classification. 65N15, 65N30, 76D07.

Received October 22, 2014. Revised March 31, 2015.

Published online January 28, 2016.

\section{INTRODUCTION}

In incompressible flows with vanishing normal velocities at the boundary, irrotational forces in the momentum equations are balanced completely by the pressure gradient. Unfortunately, nearly all mixed discretisations $[3,22]$ for the incompressible Navier-Stokes equations in the primal variables velocity and pressure do not preserve this property exactly which excites in some flow problems the so-called numerical instability of poor mass conservation $[13,19,21,30,35]$. However, poor mass conservation is just the unwanted companion of an idea which is usually regarded as a success story: the relaxation of the divergence constraint in mixed methods for

\footnotetext{
Keywords and phrases. Mixed finite element methods, incompressible Stokes problem, divergence-free methods, conforming and nonconforming FEM, mass conservation.

1 Weierstraß-Institut, Mohrenstraße 39, 10117 Berlin, Germany.

2 Institut für Numerische Mathematik, Technische Universität Dresden, 01062 Dresden, Germany. gunar.matthies@tu-dresden.de

3 Institut für Analysis und Numerik, Otto-von-Guericke-Universität Magdeburg, Universitätsplatz 2, 39106 Magdeburg, Germany.
} 
incompressible flows. This numerical instability is traditionally mitigated by stabilisation techniques like the grad-div stabilisation $[4,18,26,37,38,40]$ and does not appear for exactly divergence-free velocity approximations. A well-known example of an inf-sup stable mixed method in which discretely divergence-free velocity fields are point-wise divergence-free is the Scott-Vogelius element [41], however, it requires some restrictions on the mesh family. Recently, some ways have been achieved to overcome these mesh restrictions, each of them has its own price. For higher order polynomial approximations exactly divergence-free elements have been constructed in [16] imposing $\mathrm{C}^{1}$ regularity of velocity and pressure at the vertices of the triangulation. Suitable also for low order elements is the enrichment of the velocity space by rational divergence-free functions [23, 24]. Using dG-approximations for the tangential components of the vector Laplacian, $\mathbf{H}$ (div)-conforming divergence-free methods can be constructed $[11,27,29,42]$. Furthermore, isogeometric analysis allows to define robust mixed methods with pressure-independent velocity errors [10,15].

In order to allow to work with well-established and well-understood classical mixed methods, we follow, however, a different novel strategy which was recently proposed in [31]. It improves the robustness of such methods considerably though modifying them only slightly. The key observation is that stabilisation issues in inf-sup stable mixed methods arise only due to a relaxation of the divergence constraint in the discrete velocity test functions, and not in the trial functions. Moreover, instabilities can be traced back to those forces in the momentum balance which may possess a nontrivial irrotational part in the sense of the Helmholtz decomposition.

In the easiest case of the incompressible Stokes equations, $-\nu \Delta \mathbf{u}+\nabla p=\mathbf{f}$, $\operatorname{div} \mathbf{u}=0$, where $\nu$ and $\mathbf{f}$ denote the kinematic viscosity and a body force, poor mass conservation can only be triggered by the discretisation of the body force $\mathbf{f}$, see [31]. The novel stabilisation strategy in [31] consists in a simple replacement of the Galerkin discretisation $\int_{\Omega} \mathbf{f} \cdot \mathbf{v}_{h} \mathrm{~d} x$ by a variational crime $\int_{\Omega} \mathbf{f} \cdot \Pi_{h} \mathbf{v}_{h} \mathrm{~d} x$ where $\Pi_{h}$ is a velocity reconstruction operator. This velocity reconstruction operator has to fulfil two properties: (i) $\Pi_{h} \mathbf{v}_{h}$ has to be near to $\mathbf{v}_{h}$, and (ii) $\Pi_{h}$ has to map discretely divergence-free velocity test functions to divergence-free ones in the sense of $\mathbf{H}(\operatorname{div} ; \Omega)$. The main difference between $\mathbf{v}_{h}$ and $\Pi_{h} \mathbf{v}_{h}$ is that for discretely divergence-free test function $\mathbf{v}_{h}$ it holds $\int_{\Omega} \nabla \psi \cdot \Pi_{h} \mathbf{v}_{h} \mathrm{~d} x=0$ for all $\psi \in \mathrm{C}_{0}^{\infty}(\Omega)$ while for standard mixed methods it only holds $\int_{\Omega} \nabla \psi \cdot \mathbf{v}_{h} \mathrm{~d} x=$ $\mathcal{O}\left(h^{l+1}\right)|\psi|_{l+1}\left|\mathbf{v}_{h}\right|_{1, h}$ where $l$ denotes the approximation order of the discrete pressure space and $|\cdot|_{1, h}$ is a (maybe discrete) gradient semi-norm. Note that only the right-hand side of the discretisation is modified, but not the stiffness matrix.

In reference [31], the theoretical background for using velocity reconstruction operators was presented. This idea was applied to the first order approximation by the nonconforming Crouzeix-Raviart element [12] in two and three space dimensions. The corresponding velocity reconstruction operator maps nonconforming CrouzeixRaviart velocity test functions to lowest-order Raviart-Thomas vector fields, lifting normal velocities at simplex faces to vector fields inside of the elements. It was shown that the modified Crouzeix-Raviart element allows for a pressure-independent a priori velocity error estimate for the incompressible Stokes equations in the form

$$
\left\|\mathbf{u}-\mathbf{u}_{h}\right\|_{1, h} \leq \widetilde{C}_{\mathbf{u}} h|\mathbf{u}|_{2}
$$

while the classical Crouzeix-Raviart element [12] delivers

$$
\left\|\mathbf{u}-\mathbf{u}_{h}\right\|_{1, h} \leq C_{\mathbf{u}} h|\mathbf{u}|_{2}+\frac{C_{p}}{\nu} h|p|_{1}
$$

which predicts large velocity errors whenever $|p|_{1}$ is large or $\nu$ is small. We remark that the constant $\widetilde{C}_{\mathbf{u}}$ is slightly larger than $C_{\mathbf{u}}$ due to the additional consistency error by the variational crime [31]. It may be noted that the first mixed finite element on unstructured 3d grids for the incompressible Stokes equations that allows for pressure-independent a priori velocity error estimates was only published in 2005 [43]. Therefore, velocity reconstruction operators seem to have the potential to significantly simplify the construction of more robust, pressure-independent velocity approximations.

In this contribution, we demonstrate that this promise is indeed true. We extend the idea of using velocity reconstruction operators [31] to both nonconforming and conforming mixed finite elements of arbitrary order 
in two and three space dimensions $[3,7,22,33,34]$. Furthermore, generalising the ideas in $[1,32]$ we construct new families of nonconforming mixed finite elements of arbitrary order on rectangular and brick meshes suitable for velocity reconstruction operators. In all considered cases, the velocity reconstruction operator on simplicial meshes maps into finite element spaces of Raviart-Thomas type [36,39] and on rectangular and brick meshes into finite element spaces of Brezzi-Douglas-Marini type $[2,8,9]$. The new discretisation leads to optimal pressureindependent velocity error estimates in the discrete energy norm. Optimality is also proved for the velocity and pressure errors in the corresponding $\mathrm{L}^{2}$ norms. We emphasise that in [31] no proof for the optimality of the velocity $\mathrm{L}^{2}$ error was given which is actually a nontrivial task for this first order method. Finally, we present some numerical results confirming the theoretical predictions.

Notation. Throughout this paper, $C$ will denote a generic positive constant which is independent of the mesh size. The Stokes problem will be considered in the domain $\Omega \subset \mathbb{R}^{d}$ which is assumed to be a polygonal $(d=2)$ or polyhedral $(d=3)$ domain with boundary $\Gamma=\partial \Omega$. For a measurable $d$-dimensional subset $G$ of $\Omega$, the usual Sobolev spaces $\mathrm{W}^{m, p}(G)$ with norm $\|\cdot\|_{m, p, G}$ and semi-norm $|\cdot|_{m, p, G}$ are used. In the case $p=2$, we have $\mathrm{H}^{m}(G)=\mathrm{W}^{m, 2}(G)$ and the index $p$ will be omitted. The $\mathrm{L}^{2}$-inner product on $G$ is denoted by $(\cdot, \cdot)_{G}$. Note that the index $G$ will be omitted for $G=\Omega$. This notation of norms, semi-norms, and inner products is also used for the vector-valued and tensor-valued cases. For a sufficiently regular $(d-1)$-dimensional manifold $E \subset \partial G$ the $\mathrm{L}^{2}$-inner product on $E$ will be denoted by $\langle\cdot, \cdot\rangle_{E}$.

We will denote by $P_{k}(K)$ the space of all polynomials on $K$ with degree less than or equal to $k$ while $Q_{k}(K)$ is the space of all polynomials on $K$ with degree less than or equal to $k$ in each variable separately. For non-negative integers $\alpha$, let $L_{\alpha}$ denote the Legendre polynomials on $(-1,+1)$ which are normalised to $L_{\alpha}(1)=1$.

\section{Stokes PROBLEM AND ITS DiscRetisAtion}

\subsection{Weak formulation}

We consider the Stokes's problem

$$
-\nu \Delta \mathbf{u}+\nabla p=\mathbf{f} \quad \text { in } \Omega, \quad \operatorname{div} \mathbf{u}=0 \quad \text { in } \Omega, \quad \mathbf{u}=\mathbf{0} \quad \text { on } \Gamma,
$$

where $\mathbf{f}$ is a given body force, $\nu$ is the viscosity, $\mathbf{u}$ and $p$ denote the velocity and pressure fields, respectively. Note that for simplicity of notation we restrict ourselves to homogeneous Dirichlet boundary conditions. The extension to non-homogeneous boundary conditions is straightforward. In references [6,28], numerical examples are presented for this situation. Introducing the spaces $\mathbf{V}:=\mathrm{H}_{0}^{1}(\Omega)^{d}$ and $Q:=\mathrm{L}_{0}^{2}(\Omega)$, a weak formulation of problem (2.1) reads:

Find $(\mathbf{u}, p) \in \mathbf{V} \times Q$ such that

$$
\nu(\nabla \mathbf{u}, \nabla \mathbf{v})-(p, \operatorname{div} \mathbf{v})+(q, \operatorname{div} \mathbf{u})=(\mathbf{f}, \mathbf{v}) \quad \text { for all }(\mathbf{v}, q) \in \mathbf{V} \times Q .
$$

The Lax-Milgram's theorem applied to the subspace of divergence-free functions

$$
\mathbf{W}:=\{\mathbf{v} \in \mathbf{V}:(q, \operatorname{div} \mathbf{v})=0 \text { for all } q \in Q\}
$$

and the inf-sup condition

$$
\inf _{q \in Q} \sup _{\mathbf{v} \in \mathbf{V}} \frac{(q, \operatorname{div} \mathbf{v})}{\|q\|_{0}|\mathbf{v}|_{1}}>0
$$

guarantee that there is a unique solution of (2.2), see [22]. If $\mathbf{f}=\nabla \Phi$ with $\Phi \in \mathrm{H}^{1}(\Omega)$, we get from (2.2) by setting $q=0, \mathbf{v}=\mathbf{u} \in \mathbf{W}$

$$
\nu(\nabla \mathbf{u}, \nabla \mathbf{u})=(\nabla \Phi, \mathbf{u})=-(\Phi, \operatorname{div} \mathbf{u})=0 \quad \Longrightarrow \quad \mathbf{u}=\mathbf{0} .
$$

This means that an irrotational volume force is completely absorbed by the pressure. 
Remark 2.1. In case of non-homogeneous Dirichlet boundary conditions this property has to be reinterpreted as follows: changing the right-hand-side $\mathbf{f} \rightarrow \mathbf{f}+\nabla \psi$ with $\psi \in \mathrm{H}^{1}(\Omega) \cap \mathrm{L}_{0}^{2}(\Omega)$ changes for the continuous incompressible Stokes equations the solution by $(\mathbf{u}, p) \rightarrow(\mathbf{u}, p+\psi)$. Again, the additional gradient $\nabla \psi$ is completely absorbed by the pressure gradient. In reference [31], this property was called the 'fundamental invariance property' of the incompressible Navier-Stokes equations to indicate that it holds even for the NavierStokes equation with non-homogeneous Dirichlet boundary conditions. One should note that this fundamental invariance is also fulfilled for the generalised divergence constraint $-\nabla \cdot \mathbf{u}=g$ with $g \in \mathrm{L}_{0}^{2}(\Omega)$. In fact, this property holds for all types of boundary conditions which do not involve the pressure.

\subsection{Discrete problem and modified formulation}

We are given a family $\mathcal{T}_{h}$ of shape-regular decompositions of $\Omega$ into $d$-simplices, axiparallel quadrilaterals, or bricks. The diameter of a cell $K$ is denoted by $h_{K}$. The mesh parameter $h$ describes the maximum diameter of the cells $K \in \mathcal{T}_{h}$.

The global finite element spaces associated with the decomposition $\mathcal{T}_{h}$ are given for $k \geq 1$ by

$$
\begin{aligned}
& P_{k}^{\text {disc }}:=\left\{v \in \mathrm{L}^{2}(\Omega):\left.v\right|_{K} \in P_{k}(K) \text { for all } K \in \mathcal{T}_{h}\right\}, \quad P_{k}:=P_{k}^{\text {disc }} \cap \mathrm{H}^{1}(\Omega), \\
& Q_{k}^{\text {disc }}:=\left\{v \in \mathrm{L}^{2}(\Omega):\left.v\right|_{K} \in Q_{k}(K) \text { for all } K \in \mathcal{T}_{h}\right\}, \quad Q_{k}:=Q_{k}^{\text {disc }} \cap \mathrm{H}^{1}(\Omega) .
\end{aligned}
$$

Let $\left(\mathbf{V}_{h}, Q_{h}\right)$ be a pair of conforming or nonconforming finite element spaces over $\mathcal{T}_{h}$ approximating velocity and pressure. As usual, we will write shortly $\mathbf{V}_{h}=Q_{k}$ and $Q_{h}=P_{k}$ instead of $\mathbf{V}_{h}=\left(Q_{k} \cap \mathrm{H}_{0}^{1}(\Omega)\right)^{d}$ and $Q_{h}=\left(P_{k} \cap \mathrm{L}_{0}^{2}(\Omega)\right)$, respectively. In this paper we will consider only higher order discretisations and fix the pressure space to be $Q_{h}=P_{k-1}^{\text {disc }}$ for a fixed $k \geq 2$. The case of first order approximation has been already studied in [31]. We allow nonconforming finite element spaces $\mathbf{V}_{h}$ which are subspaces of $P_{k^{\prime}}^{\text {disc }}$ and $Q_{k^{\prime}}^{\text {disc }}$ for some $k^{\prime} \geq k$, respectively, but not of $\mathrm{H}^{1}(\Omega)$. Such functions belong to the broken $\mathrm{H}^{1}$ space

$$
\widetilde{\mathrm{H}}^{1}(\Omega):=\left\{v \in \mathrm{L}^{2}(\Omega):\left.v\right|_{K} \in \mathrm{H}^{1}(K) \text { for all } K \in \mathcal{T}_{h}\right\}
$$

which is equipped with the broken $\mathrm{H}^{1}$-semi-norm

$$
|v|_{1, h}:=\left(\sum_{K \in \mathcal{T}_{h}}|v|_{1, K}^{2}\right)^{1 / 2} .
$$

We define the piece-wise gradient $\nabla_{h}: \widetilde{\mathrm{H}}^{1}(\Omega) \rightarrow\left(\mathrm{L}^{2}(\Omega)\right)^{d}$ by $\left.\left(\nabla_{h} v\right)\right|_{K}=\nabla\left(\left.v\right|_{K}\right)$ and similar the piece-wise divergence $\operatorname{div}_{h}: \widetilde{\mathrm{H}}^{1}(\Omega)^{d} \rightarrow \mathrm{L}^{2}(\Omega)$ by $\left.\left(\operatorname{div}_{h} \mathbf{v}\right)\right|_{K}=\operatorname{div}\left(\left.\mathbf{v}\right|_{K}\right)$. Note that these definitions coincide on the spaces $\mathrm{H}^{1}(\Omega)$ and $\mathrm{H}^{1}(\Omega)^{d}$ with the standard gradient and divergence, respectively.

The set of all inner element faces $E \not \subset \partial \Omega$ of $\mathcal{T}_{h}$ will be denoted by $\mathcal{E}_{h}$. The diameter of a face $E \in \mathcal{E}_{h}$ is given by $h_{E}$. We fix for $E \in \mathcal{E}_{h}$ a unit normal vector $\mathbf{n}_{E}$. The two cells which share $E$ are denoted by $K_{E}$ and $K_{E}^{\prime}$ such that $\mathbf{n}_{E}$ points from $K_{E}$ into $K_{E}^{\prime}$. We define for a piece-wise smooth function $r_{h}$ its jump over the face $E$ as

$$
\left[r_{h}\right]_{E}:=\left.\left(\left.r_{h}\right|_{K_{E}}\right)\right|_{E}-\left.\left(\left.r_{h}\right|_{K_{E}^{\prime}}\right)\right|_{E} .
$$

We set $\mathbf{n}_{E}=\mathbf{n}$ for all element faces $E \subset \Gamma$ on the boundary where $\mathbf{n}$ is the outer unit normal to $\Omega$.

In order to guarantee existence, uniqueness, and accuracy of solutions of the discrete problem we make the following abstract assumptions. We consider in Section 4 finite element spaces satisfying all the assumptions made here.

Assumption A1. The pair $\left(\mathbf{V}_{h}, Q_{h}\right)$ fulfils the discrete inf-sup condition, i.e., there exists a positive constant $\beta$ such that

$$
\inf _{q_{h} \in Q_{h}} \sup _{\mathbf{v}_{h} \in \mathbf{V}_{h}} \frac{\left(q_{h}, \operatorname{div}_{h} \mathbf{v}_{h}\right)}{\left\|q_{h}\right\|_{0}\left|\mathbf{v}_{h}\right|_{1, h}} \geq \beta>0
$$

holds uniformly in $h$. 
Assumption A2. There exists an integer $r \geq k-1$ such that

$$
\left\langle q,\left[\mathbf{v}_{h}\right]\right\rangle_{E}=\mathbf{0} \quad \text { for all } q \in P_{r}(E), E \in \mathcal{E}_{h}, \quad\left\langle q, \mathbf{v}_{h}\right\rangle_{E}=\mathbf{0} \quad \text { for all } q \in P_{r}(E), E \subset \Gamma
$$

holds for all $\mathbf{v}_{h} \in \mathbf{V}_{h}$.

Remark 2.2. Assumption A2 guarantees that the broken $\mathrm{H}^{1}$-semi-norm $|\cdot|_{1, h}$ is a norm on $\mathbf{V}+\mathbf{V}_{h}$, see [12]. Assumption A2 is satisfied for any conforming finite element space $\mathbf{V}_{h} \subset \mathbf{V}$.

Assumption A3. The finite element space $\mathbf{V}_{h}$ approximates $\mathbf{V}$ of order $k$, i.e., there exists an interpolation operator $i_{h}: \mathbf{V} \cap \mathrm{H}^{k+1}(\Omega)^{d} \rightarrow \mathbf{V}_{h}$ such that

$$
\left\|\mathbf{v}-i_{h} \mathbf{v}\right\|_{0, K}+h_{K}\left|\mathbf{v}-i_{h} \mathbf{v}\right|_{1, K} \leq C h_{K}^{k+1}|\mathbf{v}|_{k+1, K} \quad \text { for all } \mathbf{v} \in \mathrm{H}^{k+1}(K)^{d}, K \in \mathcal{T}_{h} .
$$

Remark 2.3. The local $\mathrm{L}^{2}$-projections $j_{h}: \mathrm{L}^{2}(K) \rightarrow P_{k-1}(K), K \in \mathcal{T}_{h}$, satisfy

$$
\left\|w-j_{h} w\right\|_{0, K}+h_{K}\left|w-j_{h} w\right|_{1, K} \leq C h_{K}^{k}|w|_{k, K} \quad \text { for all } w \in \mathrm{H}^{k}(K)
$$

and will sometimes be combined to the global $\mathrm{L}^{2}$-projection $j_{h}: \mathrm{L}^{2}(\Omega) \rightarrow P_{k-1}^{\text {disc }}$.

Now, the standard Galerkin discretisation reads:

Find $\left(\mathbf{u}_{h}, p_{h}\right) \in \mathbf{V}_{h} \times Q_{h}$ such that

$$
\nu\left(\nabla_{h} \mathbf{u}_{h}, \nabla_{h} \mathbf{v}_{h}\right)-\left(p_{h}, \operatorname{div}_{h} \mathbf{v}_{h}\right)+\left(q_{h}, \operatorname{div}_{h} \mathbf{u}_{h}\right)=\left(\mathbf{f}, \mathbf{v}_{h}\right)
$$

for all $\left(\mathbf{v}_{h}, q_{h}\right) \in \mathbf{V}_{h} \times Q_{h}$. As in the continuous case, the Lax-Milgram theorem can be applied to the subspace of discretely divergence-free functions

$$
\mathbf{W}_{h}:=\left\{\mathbf{v}_{h} \in \mathbf{V}_{h}:\left(q_{h}, \operatorname{div}_{h} \mathbf{v}_{h}\right)=0 \text { for all } q_{h} \in Q_{h}\right\}
$$

which leads to the unique solvability of the pressure-free formulation of the discrete Stokes problem:

$$
\text { Find } \mathbf{u}_{h} \in \mathbf{W}_{h} \text { such that } \quad \nu\left(\nabla_{h} \mathbf{u}_{h}, \nabla_{h} \mathbf{v}_{h}\right)=\left(\mathbf{f}, \mathbf{v}_{h}\right) \text { for all } \mathbf{v}_{h} \in \mathbf{W}_{h} .
$$

The existence of a unique pressure $p_{h} \in Q_{h}$ such that $\left(\mathbf{u}_{h}, p_{h}\right) \in \mathbf{V}_{h} \times Q_{h}$ solves (2.9) follows from Assumption A1.

Remark 2.4. If again $\mathbf{f}=\nabla \Phi$ with $\Phi \in \mathrm{H}^{1}(\Omega)$, we get from (2.11) by setting $\mathbf{v}_{h}=\mathbf{u}_{h} \in \mathbf{W}_{h}$

$$
\nu\left(\nabla_{h} \mathbf{u}_{h}, \nabla_{h} \mathbf{u}_{h}\right)=\left(\nabla \Phi, \mathbf{u}_{h}\right)=\sum_{K \in \mathcal{T}_{h}}\left\{\left\langle\Phi, \mathbf{u}_{h} \cdot \mathbf{n}_{K}\right\rangle_{\partial K}-\left(\Phi, \operatorname{div} \mathbf{u}_{h}\right)_{K}\right\} \neq 0,
$$

in general. Note that even in the case of a conforming method, we have

$$
\nu\left(\nabla \mathbf{u}_{h}, \nabla \mathbf{u}_{h}\right)=-\left(\Phi, \operatorname{div} \mathbf{u}_{h}\right) \neq 0,
$$

since $\Phi \notin Q_{h}$ in general.

Following [31], our aim is to modify the finite element method in such a way that the discrete velocity solution for $\mathbf{f}=\nabla \Phi$ becomes $\mathbf{u}_{h}=0$.

We introduce the spaces

$$
\mathbf{X}:=\mathbf{H}(\operatorname{div} ; \Omega)=\left\{\mathbf{v} \in \mathrm{L}^{2}(\Omega)^{d}: \operatorname{div} \mathbf{v} \in \mathrm{L}^{2}(\Omega)\right\}, \quad \mathbf{X}^{0}:=\{\mathbf{v} \in \mathbf{X}: \operatorname{div} \mathbf{v}=0\},
$$

and recall that functions in $\mathbf{X}$ allow normal traces. In particular, we have Green's formula ([3], Lem. 2.1.1). 
Lemma 2.5. For $\mathbf{v} \in \mathbf{H}(\operatorname{div} ; \Omega)$ we can define $\mathbf{v} \cdot \mathbf{n} \in \mathrm{H}^{-1 / 2}(\Gamma)$ such that

$$
(\operatorname{div} \mathbf{v}, q)=\langle\mathbf{v} \cdot \mathbf{n}, q\rangle_{\Gamma}-(\mathbf{v}, \nabla q) \quad \text { for all } q \in \mathrm{H}^{1}(\Omega) .
$$

Assumption A4. There exist an integer $s \geq k-1$ and a finite element space $\mathbf{X}_{h} \subset \mathbf{X}$ such that

$$
\begin{aligned}
\left.\mathbf{v}_{h} \cdot \mathbf{n}_{E}\right|_{E} \in P_{s}(E) & \text { for all } \mathbf{v}_{h} \in \mathbf{X}_{h}, E \subset \partial K, K \in \mathcal{T}_{h}, \\
\left.\operatorname{div}_{h} \mathbf{v}_{h}\right|_{K} \in P_{k-1}(K) & \text { for all } \mathbf{v}_{h} \in \mathbf{X}_{h}, K \in \mathcal{T}_{h}, \\
\left\langle q,\left[\mathbf{v}_{h} \cdot \mathbf{n}_{E}\right]\right\rangle_{E}=0 & \text { for all } q \in P_{s}(E), E \in \mathcal{E}_{h}, \mathbf{v}_{h} \in \mathbf{X}_{h}, \\
\left\langle q, \mathbf{v}_{h} \cdot \mathbf{n}\right\rangle_{E}=0 & \text { for all } q \in P_{s}(E), E \subset \Gamma, \mathbf{v}_{h} \in \mathbf{X}_{h} .
\end{aligned}
$$

Further, we assume that there is an operator $\Pi_{h}: \mathbf{V}+\mathbf{V}_{h} \rightarrow \mathbf{X}_{h}$ with

$$
\begin{aligned}
&\left(\mathbf{v}-\Pi_{h} \mathbf{v}, \mathbf{w}\right)_{K}=0 \quad \text { for all } \mathbf{v} \in \mathbf{V}+\mathbf{V}_{h}, \mathbf{w} \in P_{k-2}(K)^{d}, K \in \mathcal{T}_{h} \\
&\left\langle\left(\mathbf{v}-\Pi_{h} \mathbf{v}\right) \cdot \mathbf{n}_{E}, q\right\rangle_{E}=0 \quad \text { for all } \mathbf{v} \in \mathbf{V}+\mathbf{V}_{h}, q \in P_{s}(E), E \subset \partial K, K \in \mathcal{T}_{h} \\
&\left\|\Pi_{h} \mathbf{v}-\mathbf{v}\right\|_{0, K} \leq C h_{K}^{m}|\mathbf{v}|_{m, K} \quad \text { for all } \mathbf{v} \in \mathbf{V}+\mathbf{V}_{h}, K \in \mathcal{T}_{h}, m=0,1,2
\end{aligned}
$$

We will see later in Section 4 that the family of Raviart-Thomas spaces $\mathbf{R T}_{k-1}$ for $s=k-1$ on simplicial meshes and the family of Brezzi-Douglas-Marini's spaces $\mathbf{B D M}_{k}$ for $s=k$ on rectangular and brick meshes satisfy Assumption A4. The extension to the general case of quadrilaterals and hexahedra requires additional technical tools as the Piola transformation for non-affine mappings and a careful study of the approximation properties of Brezzi-Douglas-Marini spaces on general quadrilateral and hexahedral meshes. In this paper, we restrict ourselves to families of rectangular and brick meshes to avoid that the main ideas are hidden behind technical details. The general case will be the subject of a forthcoming paper.

Following the idea in [31], we consider the modified discrete problem

Find $\left(\mathbf{u}_{h}, p_{h}\right) \in \mathbf{V}_{h} \times Q_{h}$ such that for all $\left(\mathbf{v}_{h}, q_{h}\right) \in \mathbf{V}_{h} \times Q_{h}$

$$
\nu\left(\nabla_{h} \mathbf{u}_{h}, \nabla_{h} \mathbf{v}_{h}\right)-\left(p_{h}, \operatorname{div}_{h} \mathbf{v}_{h}\right)+\left(q_{h}, \operatorname{div}_{h} \mathbf{u}_{h}\right)=\left(\mathbf{f}, \Pi_{h} \mathbf{v}_{h}\right) .
$$

Note that the force term $\mathbf{f}$ has to be slightly more regular compared to (2.2). It has to belong now to the dual space of $\mathbf{H}(\operatorname{div} ; \Omega)$.

\section{ERror estimates}

First we show that the operator $\Pi_{h}$ maps discretely divergence-free functions into divergence-free functions in the sense of $\mathbf{H}(\operatorname{div} ; \Omega)$. Moreover, the normal component on the domain boundary $\Gamma$ vanishes in a strong sense.

Lemma 3.1. Suppose A2 and A4 with $r \geq s \geq k-1 \geq 1$. Then, we have

$$
\left(q_{h}, \operatorname{div} \Pi_{h} \mathbf{v}_{h}\right)=\left(q_{h}, \operatorname{div}_{h} \mathbf{v}_{h}\right)
$$

for all $\mathbf{v}_{h} \in \mathbf{V}_{h}$ and for all $q_{h} \in Q_{h}=P_{k-1}^{\text {disc }}$. Furthermore, it holds $\Pi_{h}\left(\mathbf{W}_{h}\right) \subset \mathbf{X}^{0}$ and $\Pi_{h} \mathbf{v} \cdot \mathbf{n}=0$ on $\Gamma$ for all $\mathbf{v} \in \mathbf{V}+\mathbf{V}_{h}$.

Proof. We obtain for all $q_{h} \in Q_{h}=P_{k-1}^{\text {disc }}$ and all $\mathbf{v}_{h} \in \mathbf{V}_{h}$ by element-wise integration by parts

$$
\left(q_{h}, \operatorname{div} \Pi_{h} \mathbf{v}_{h}\right)=\sum_{K \in \mathcal{T}_{h}}\left(q_{h}, \operatorname{div} \Pi_{h} \mathbf{v}_{h}\right)_{K}=\sum_{K \in \mathcal{T}_{h}}\left\{\left\langle q_{h}, \Pi_{h} \mathbf{v}_{h} \cdot \mathbf{n}_{K}\right\rangle_{\partial K}-\left(\nabla q_{h}, \Pi_{h} \mathbf{v}_{h}\right)_{K}\right\}
$$


where $\mathbf{n}_{K}$ denotes the outward unit normal vector to $K$. Taking into consideration that $\left.q_{h}\right|_{E} \in P_{k-1}(E)$ and $\left.\nabla q_{h}\right|_{K} \in P_{k-2}(K)^{d}$, and applying (2.17) and (2.18), we have

$$
\left(q_{h}, \operatorname{div} \Pi_{h} \mathbf{v}_{h}\right)=\sum_{K \in \mathcal{T}_{h}}\left\{\left\langle q_{h}, \mathbf{v}_{h} \cdot \mathbf{n}_{K}\right\rangle_{\partial K}-\left(\nabla q_{h}, \mathbf{v}_{h}\right)_{K}\right\}=\sum_{K \in \mathcal{T}_{h}}\left(q_{h}, \operatorname{div} \mathbf{v}_{h}\right)_{K}=\left(q_{h}, \operatorname{div}_{h} \mathbf{v}_{h}\right)
$$

for all $q_{h} \in Q_{h}$. Hence, we obtain for $\mathbf{v}_{h} \in \mathbf{W}_{h}$ that $\left(q_{h}, \operatorname{div} \Pi_{h} \mathbf{v}_{h}\right)=0$ for all $q_{h} \in Q_{h}=P_{k-1}^{\text {disc }}$. Due to $\operatorname{div} \Pi_{h} \mathbf{v}_{h} \in P_{k-1}^{\text {disc }}$ we can set $q_{h}=\operatorname{div} \Pi_{h} \mathbf{v}_{h}$ to get $\operatorname{div} \Pi_{h} \mathbf{v}_{h}=0$ in the sense of $\mathbf{H}(\operatorname{div} ; \Omega)$.

We obtain from (2.18) and (2.6) for all $E \subset \Gamma$, all $\mathbf{v} \in \mathbf{V}+\mathbf{V}_{h}$, and all $q \in P_{s}(E)$ with $r \geq s$

$$
\left\langle\Pi_{h} \mathbf{v} \cdot \mathbf{n}_{E}, q\right\rangle_{E}=\left\langle\mathbf{v} \cdot \mathbf{n}_{E}, q\right\rangle_{E}=\langle\mathbf{v}, q\rangle_{E} \cdot \mathbf{n}_{E}=0 .
$$

Due to $\Pi_{h} \mathbf{v} \cdot \mathbf{n}_{E} \in P_{s}(E)$ we can set $q=\Pi_{h} \mathbf{v} \cdot \mathbf{n}_{E}$ to get $\Pi_{h} \mathbf{v} \cdot \mathbf{n}_{E}=0$ on $E \subset \Gamma$.

Lemma 3.2. Suppose $\mathrm{A} 2$ and $\mathrm{A} 4$ for $r \geq k-1 \geq 1$. Let $\mathbf{v} \in \mathbf{V}+\mathbf{V}_{h}$. For $\mathbf{u} \in \mathrm{H}^{k+1}(\Omega)^{d}$, we have

$$
\left|\left(\Delta \mathbf{u}, \Pi_{h} \mathbf{v}\right)+\left(\nabla \mathbf{u}, \nabla_{h} \mathbf{v}\right)\right| \leq C \sum_{K \in \mathcal{T}_{h}} h_{K}^{k}|\mathbf{u}|_{k+1, K}|\mathbf{v}|_{1, K}
$$

while

$$
\left|\left(\nabla p, \Pi_{h} \mathbf{v}\right)+\left(p, \operatorname{div}_{h} \mathbf{v}\right)\right| \leq C \sum_{K \in \mathcal{T}_{h}} h_{K}^{k}|p|_{k, K}|\mathbf{v}|_{1, K}
$$

holds true for $p \in \mathrm{H}^{k}(\Omega)$.

Proof. We add and subtract $(\Delta \mathbf{u}, \mathbf{v})$ to get

$$
\left(\Delta \mathbf{u}, \Pi_{h} \mathbf{v}\right)+\left(\nabla \mathbf{u}, \nabla_{h} \mathbf{v}\right)=\left(\Delta \mathbf{u}, \Pi_{h} \mathbf{v}-\mathbf{v}\right)+\left(\nabla \mathbf{u}, \nabla_{h} \mathbf{v}\right)+(\Delta \mathbf{u}, \mathbf{v}) .
$$

The property (2.17) of $\Pi_{h}: \mathbf{V}+\mathbf{V}_{h} \rightarrow \mathbf{X}_{h}$ allows to subtract the local L $\mathrm{L}^{2}$-projection $\pi_{k-2}^{K}: \mathrm{L}^{2}(K) \rightarrow P_{k-2}(K)$. We use (2.19) to estimate the first term in (3.3) as follows

$$
\begin{aligned}
\left|\left(\Delta \mathbf{u}, \Pi_{h} \mathbf{v}-\mathbf{v}\right)\right| & =\left|\sum_{K \in \mathcal{T}_{h}}\left(\Delta \mathbf{u}-\pi_{k-2}^{K} \Delta \mathbf{u}, \Pi_{h} \mathbf{v}-\mathbf{v}\right)_{K}\right| \leq C \sum_{K \in \mathcal{T}_{h}} h_{K}^{k-1}|\Delta \mathbf{u}|_{k-1, K} h_{K}|\mathbf{v}|_{1, K} \\
& \leq C \sum_{K \in \mathcal{T}_{h}} h_{K}^{k}|\mathbf{u}|_{k+1, K}|\mathbf{v}|_{1, K}
\end{aligned}
$$

The sum of the second and third term in (3.3) is related to the consistency error of the nonconforming method. We integrate as in [12] element-wise by parts

$$
\left(\nabla \mathbf{u}, \nabla_{h} \mathbf{v}\right)+(\Delta \mathbf{u}, \mathbf{v})=\sum_{K \in \mathcal{T}_{h}}\left\langle\frac{\partial \mathbf{u}}{\partial \mathbf{n}_{K}}, \mathbf{v}\right\rangle_{\partial K}=\sum_{E \in \mathcal{E}_{h}}\left\langle\frac{\partial \mathbf{u}}{\partial \mathbf{n}_{E}},[\mathbf{v}]_{E}\right\rangle_{E}+\sum_{E \subset \Gamma}\left\langle\frac{\partial \mathbf{u}}{\partial \mathbf{n}_{E}}, \mathbf{v}\right\rangle_{E},
$$

use A2, insert the $\mathrm{L}^{2}$-projection $\pi_{k-1}^{E}: \mathrm{L}^{2}(E) \rightarrow P_{k-1}(E)$, and apply the estimate from Lemma 3 in [12] to obtain

$$
\left|\left(\nabla \mathbf{u}, \nabla_{h} \mathbf{v}\right)+(\Delta \mathbf{u}, \mathbf{v})\right|=\left|\sum_{K \in \mathcal{T}_{h}} \sum_{E \subset \partial K}\left\langle\frac{\partial \mathbf{u}}{\partial \mathbf{n}_{E}}-\pi_{k-1}^{E} \frac{\partial \mathbf{u}}{\partial \mathbf{n}_{E}}, \mathbf{v}\right\rangle_{E}\right| \leq C \sum_{K \in \mathcal{T}_{h}} h_{K}^{k}|\mathbf{u}|_{k+1, K}|\mathbf{v}|_{1, K} .
$$


For proving (3.2), we first add and subtract $(\nabla p, \mathbf{v})$ to have

$$
\left(\nabla p, \Pi_{h} \mathbf{v}\right)+\left(p, \operatorname{div}_{h} \mathbf{v}\right)=\left(\nabla p, \Pi_{h} \mathbf{v}-\mathbf{v}\right)+\left(p, \operatorname{div}_{h} \mathbf{v}\right)+(\nabla p, \mathbf{v}) .
$$

In the first term, we subtract the local $\mathrm{L}^{2}$-projection $\pi_{k-2}^{K}: \mathrm{L}^{2}(K) \rightarrow P_{k-2}(K)$ resulting in

$$
\begin{aligned}
\left|\left(\nabla p, \Pi_{h} \mathbf{v}-\mathbf{v}\right)\right| & =\left|\sum_{K \in \mathcal{T}_{h}}\left(\nabla p-\pi_{k-2}^{K} \nabla p, \Pi_{h} \mathbf{v}-\mathbf{v}\right)_{K}\right| \leq C \sum_{K \in \mathcal{T}_{h}} h_{K}^{k-1}|\nabla p|_{k-1, K} h_{K}|\mathbf{v}|_{1, K} \\
& \leq C \sum_{K \in \mathcal{T}_{h}} h_{K}^{k}|p|_{k, K}|\mathbf{v}|_{1, K} .
\end{aligned}
$$

The sum of the second and third term in (3.4) represents again the consistency error of the nonconforming method. It can be handled as in ([12], Lem. 3) by an element-wise integration by parts

$$
\left(p, \operatorname{div}_{h} \mathbf{v}\right)+(\nabla p, \mathbf{v})=\sum_{K \in \mathcal{T}_{h}}\left\langle p, \mathbf{v} \cdot \mathbf{n}_{K}\right\rangle_{\partial K}=\sum_{E \in \mathcal{E}_{h}}\left\langle p,[\mathbf{v}]_{E} \cdot \mathbf{n}_{E},\right\rangle_{E}+\sum_{E \subset \Gamma}\left\langle p, \mathbf{v} \cdot \mathbf{n}_{E}\right\rangle_{E}
$$

and using the $\mathrm{L}^{2}$-projection $\pi_{k-1}^{E}: \mathrm{L}^{2}(E) \rightarrow P_{k-1}(E)$ to get

$$
\left|\left(p, \operatorname{div}_{h} \mathbf{v}\right)+(\nabla p, \mathbf{v})\right|=\left|\sum_{K \in \mathcal{T}_{h}} \sum_{E \subset \partial K}\left\langle p-\pi_{k-1}^{E} p, \mathbf{v} \cdot \mathbf{n}_{E}\right\rangle_{E}\right| \leq C \sum_{K \in \mathcal{T}_{h}} h_{K}^{k}|p|_{k, K}|\mathbf{v}|_{1, K}
$$

Collecting all estimates, we obtain the statement of the Lemma.

Theorem 3.3. Let the solution $(\mathbf{u}, p)$ of $(2.2)$ belong to $\mathrm{H}^{2}(\Omega)^{d} \times \mathrm{H}^{1}(\Omega)$. Furthermore, $\left(\mathbf{u}_{h}, p_{h}\right) \in \mathbf{V}_{h} \times Q_{h}$ is the solution of (2.20). Let $\mathrm{A} 1-\mathrm{A} 4$ be satisfied for $r \geq s \geq k-1$. If $\mathbf{u} \in \mathrm{H}^{k+1}(\Omega)^{d}$, then the error estimates

$$
\begin{gathered}
\left|\mathbf{u}-\mathbf{u}_{h}\right|_{1, h} \leq C\left(\sum_{K \in \mathcal{T}_{h}} h_{K}^{2 k}|\mathbf{u}|_{k+1, K}^{2}\right)^{1 / 2} \\
\left\|j_{h} p-p_{h}\right\|_{0} \leq C\left(\sum_{K \in \mathcal{T}_{h}} h_{K}^{2 k} \nu^{2}|\mathbf{u}|_{k+1, K}^{2}\right)^{1 / 2}
\end{gathered}
$$

hold true. If in addition $p \in \mathrm{H}^{k}(\Omega)$, then the estimate

$$
\left\|p-p_{h}\right\|_{0} \leq C\left(\sum_{K \in \mathcal{T}_{h}} h_{K}^{2 k}\left[\nu^{2}|\mathbf{u}|_{k+1, K}^{2}+|p|_{k, K}^{2}\right]\right)^{1 / 2}
$$

is obtained.

Proof. Let $\mathbf{u}_{h}$ be the solution of the modified discrete problem (2.20) and $\mathbf{v}_{h} \in \mathbf{W}_{h}$ arbitrary. Then, we have $\mathbf{w}_{h}:=\mathbf{u}_{h}-\mathbf{v}_{h} \in \mathbf{W}_{h}$. Applying the triangle inequality

$$
\left|\mathbf{u}-\mathbf{u}_{h}\right|_{1, h} \leq\left|\mathbf{u}-\mathbf{v}_{h}\right|_{1, h}+\left|\mathbf{w}_{h}\right|_{1, h},
$$

we see that $\left|\mathbf{w}_{h}\right|_{1, h}$ has to be estimated. Since $\mathbf{u}$ and $\mathbf{u}_{h}$ are the solution of the continuous and modified discrete problem, respectively, we get

$$
\begin{aligned}
\nu\left|\mathbf{w}_{h}\right|_{1, h}^{2} & =\nu\left(\nabla_{h}\left(\mathbf{u}_{h}-\mathbf{v}_{h}\right), \nabla_{h} \mathbf{w}_{h}\right)=\nu\left(\nabla_{h}\left(\mathbf{u}-\mathbf{v}_{h}\right), \nabla_{h} \mathbf{w}_{h}\right)+\nu\left(\nabla_{h}\left(\mathbf{u}_{h}-\mathbf{u}\right), \nabla_{h} \mathbf{w}_{h}\right) \\
& =\nu\left(\nabla_{h}\left(\mathbf{u}-\mathbf{v}_{h}\right), \nabla_{h} \mathbf{w}_{h}\right)+\left(\mathbf{f}, \Pi_{h} \mathbf{w}_{h}\right)-\nu\left(\nabla \mathbf{u}, \nabla_{h} \mathbf{w}_{h}\right) .
\end{aligned}
$$


The first term on the right-hand side of (3.8) is bounded by the Cauchy-Schwarz's inequality

$$
\nu\left|\left(\nabla_{h}\left(\mathbf{u}-\mathbf{v}_{h}\right), \nabla_{h} \mathbf{w}_{h}\right)\right| \leq \nu\left|\mathbf{u}-\mathbf{v}_{h}\right|_{1, h}\left|\mathbf{w}_{h}\right|_{1, h},
$$

the second and third term can be bounded by Lemma 3.2 in the following way

$$
\begin{aligned}
\left(\mathbf{f}, \Pi_{h} \mathbf{w}_{h}\right)-\nu\left(\nabla \mathbf{u}, \nabla_{h} \mathbf{w}_{h}\right) & =\left(-\nu \Delta \mathbf{u}+\nabla p, \Pi_{h} \mathbf{w}_{h}\right)-\nu\left(\nabla \mathbf{u}, \nabla_{h} \mathbf{w}_{h}\right) \\
& =-\nu\left[\left(\Delta \mathbf{u}, \Pi_{h} \mathbf{w}_{h}\right)+\left(\nabla \mathbf{u}, \nabla_{h} \mathbf{w}_{h}\right)\right] \\
\left|\left(\mathbf{f}, \Pi_{h} \mathbf{w}_{h}\right)-\nu\left(\nabla \mathbf{u}, \nabla_{h} \mathbf{w}_{h}\right)\right| & \leq C\left(\sum_{K \in \mathcal{T}_{h}} h_{K}^{2 k} \nu^{2}|\mathbf{u}|_{k+1, K}^{2}\right)^{1 / 2}\left|\mathbf{w}_{h}\right|_{1, h} .
\end{aligned}
$$

Note that we used Green's formula and Lemma 3.1 to show that

$$
\left(\nabla p, \Pi_{h} \mathbf{w}_{h}\right)=\left\langle p, \Pi_{h} \mathbf{w}_{h} \cdot \mathbf{n}\right\rangle-\left(p, \operatorname{div} \Pi_{h} \mathbf{w}_{h}\right)=0 .
$$

Taking the infimum over all $\mathbf{v}_{h} \in \mathbf{W}_{h}$ and using the inf-sup condition as in ([22], II (1.16)), we obtain

$$
\inf _{\mathbf{v}_{h} \in \mathbf{W}_{h}}\left|\mathbf{u}-\mathbf{v}_{h}\right|_{1, h} \leq C \inf _{\mathbf{v}_{h} \in \mathbf{V}_{h}}\left|\mathbf{u}-\mathbf{v}_{h}\right|_{1, h} \leq C\left(\sum_{K \in \mathcal{T}_{h}} h_{K}^{2 k}|\mathbf{u}|_{k+1, K}^{2}\right)^{1 / 2}
$$

and end up with estimate (3.5).

In order to prove (3.6), we use the modified discrete problem (2.20) and $\mathbf{f}=-\nu \Delta \mathbf{u}+\nabla p$ to compute for $\mathbf{v}_{h} \in \mathbf{V}_{h}$

$$
\begin{aligned}
\left(j_{h} p-p_{h}, \operatorname{div}_{h} \mathbf{v}_{h}\right) & =\left(j_{h} p, \operatorname{div}_{h} \mathbf{v}_{h}\right)+\left(\mathbf{f}, \Pi_{h} \mathbf{v}_{h}\right)-\nu\left(\nabla_{h} \mathbf{u}_{h}, \nabla_{h} \mathbf{v}_{h}\right) \\
& =\left(j_{h} p, \operatorname{div}_{h} \mathbf{v}_{h}\right)+\left(\nabla p, \Pi_{h} \mathbf{v}_{h}\right)-\nu\left(\Delta \mathbf{u}, \Pi_{h} \mathbf{v}_{h}\right)-\nu\left(\nabla_{h} \mathbf{u}_{h}, \nabla_{h} \mathbf{v}_{h}\right) .
\end{aligned}
$$

We obtain for the first and the second term

$$
\left(j_{h} p, \operatorname{div}_{h} \mathbf{v}_{h}\right)+\left(\nabla p, \Pi_{h} \mathbf{v}_{h}\right)=\left(j_{h} p, \operatorname{div}_{h} \mathbf{v}_{h}\right)-\left(p, \operatorname{div} \Pi_{h} \mathbf{v}_{h}\right),
$$

by using Lemma 2.5. However, due to $\operatorname{div} \Pi_{h} \mathbf{v}_{h} \in Q_{h}$, it holds $\left(p, \operatorname{div} \Pi_{h} \mathbf{v}_{h}\right)=\left(j_{h} p, \operatorname{div} \Pi_{h} \mathbf{v}_{h}\right)$ and

$$
\left(j_{h} p, \operatorname{div}_{h} \mathbf{v}_{h}\right)+\left(\nabla p, \Pi_{h} \mathbf{v}_{h}\right)=\left(j_{h} p, \operatorname{div}_{h} \mathbf{v}_{h}-\operatorname{div} \Pi_{h} \mathbf{v}_{h}\right)=0
$$

due to Lemma 3.1. Hence, adding and subtracting $-\nu\left(\nabla \mathbf{u}, \nabla_{h} \mathbf{v}_{h}\right)$ leads to

$$
\left(j_{h} p-p_{h}, \operatorname{div}_{h} \mathbf{v}_{h}\right)=-\nu\left\{\left(\Delta \mathbf{u}, \Pi_{h} \mathbf{v}_{h}\right)+\left(\nabla \mathbf{u}, \nabla_{h} \mathbf{v}_{h}\right)\right\}+\nu\left(\nabla_{h}\left(\mathbf{u}-\mathbf{u}_{h}\right), \nabla_{h} \mathbf{v}_{h}\right) .
$$

By the discrete inf-sup stability (2.4), we get the estimate

$$
\left\|j_{h} p-p_{h}\right\|_{0} \leq \frac{1}{\beta} \sup _{\mathbf{v}_{h} \in \mathbf{V}_{h}} \frac{\left(j_{h} p-p_{h}, \operatorname{div}_{h} \mathbf{v}_{h}\right)}{\left|\mathbf{v}_{h}\right|_{1, h}} .
$$

We finally prove (3.6) using the Cauchy-Schwarz's inequality, Lemma 3.2, and (3.5).

Now, as a simple consequence of the triangle inequality

$$
\left\|p-p_{h}\right\|_{0} \leq\left\|p-j_{h} p\right\|_{0}+\left\|j_{h} p-p_{h}\right\|_{0}
$$

estimate (3.7) follows. 
Remark 3.4. The estimate (3.6) shows that not only the velocity error is pressure-independent. Also, the discrete pressure is the best approximation in the discrete pressure space, up to an error which is also pressureindependent.

Next we assume that the Stokes's problem is $\mathrm{H}^{2}(\Omega)^{d} \times \mathrm{H}^{1}(\Omega)$-regular, i.e., there exists for any $\mathbf{g} \in \mathrm{L}^{2}(\Omega)^{d}$ a unique solution $\left(\mathbf{z}_{\mathbf{g}}, r_{\mathbf{g}}\right) \in\left(\mathbf{V} \cap \mathrm{H}^{2}(\Omega)^{d}\right) \times\left(Q \cap \mathrm{H}^{1}(\Omega)\right)$ of the problem

Find $\left(\mathbf{z}_{\mathbf{g}}, r_{\mathbf{g}}\right) \in \mathbf{V} \times Q$ such that for all $(\mathbf{v}, q) \in \mathbf{V} \times Q$

$$
\nu\left(\nabla \mathbf{v}, \nabla \mathbf{z}_{\mathbf{g}}\right)-\left(r_{\mathbf{g}}, \operatorname{div} \mathbf{v}\right)+\left(q, \operatorname{div} \mathbf{z}_{\mathbf{g}}\right)=(\mathbf{g}, \mathbf{v})
$$

satisfying

$$
\nu\left|\mathbf{z}_{\mathbf{g}}\right|_{2}+\left|r_{\mathbf{g}}\right|_{1} \leq C\|\mathbf{g}\|_{0} .
$$

Theorem 3.5. Let $(\mathbf{u}, p)$ and $\left(\mathbf{u}_{h}, p_{h}\right)$ denote the solutions of (2.2) and (2.20), respectively. Furthermore, let $\mathbf{u}$ belong to $\mathrm{H}^{k+1}(\Omega)^{d}$. If $\mathrm{A} 1-\mathrm{A} 4$ are satisfied for $r \geq s \geq k-1$ and the Stokes problem is $\mathrm{H}^{2}(\Omega)^{d} \times \mathrm{H}^{1}(\Omega)$-regular, then there exists a positive constant $C$ independent of $h$ and $\nu$ such that

$$
\left\|\mathbf{u}-\mathbf{u}_{h}\right\|_{0} \leq C h^{k+1}|\mathbf{u}|_{k+1}
$$

holds.

Proof. Using $-\nu \Delta \mathbf{z}_{\mathbf{g}}+\nabla r_{\mathbf{g}}=\mathbf{g}$, integrating element-wise by parts, and taking into consideration A2, we get

$$
\begin{aligned}
\left(\mathbf{g}, \mathbf{u}-\mathbf{u}_{h}\right)= & \nu\left(\nabla_{h}\left(\mathbf{u}-\mathbf{u}_{h}\right), \nabla \mathbf{z}_{\mathbf{g}}\right)-\left(r_{\mathbf{g}}, \operatorname{div}_{h}\left(\mathbf{u}-\mathbf{u}_{h}\right)\right) \\
& -\sum_{E \in \mathcal{E}_{h}}\left\{\left\langle\nu \frac{\partial \mathbf{z}_{\mathbf{g}}}{\partial \mathbf{n}_{E}},\left[\mathbf{u}-\mathbf{u}_{h}\right]_{E}\right\rangle_{E}-\left\langle r_{\mathbf{g}},\left[\mathbf{u}-\mathbf{u}_{h}\right]_{E} \cdot \mathbf{n}_{E}\right\rangle_{E}\right\} \\
& -\sum_{E \subset \Gamma}\left\{\left\langle\nu \frac{\partial \mathbf{z}_{\mathbf{g}}}{\partial \mathbf{n}_{E}}, \mathbf{u}-\mathbf{u}_{h}\right\rangle_{E}-\left\langle r_{\mathbf{g}},\left(\mathbf{u}-\mathbf{u}_{h}\right) \cdot n_{E}\right\rangle_{E}\right\} .
\end{aligned}
$$

Choosing appropriate interpolations $\left(\mathbf{z}_{\mathbf{g}, h}, r_{\mathbf{g}, h}\right) \in \mathbf{W}_{h} \times Q_{h}$, having in mind that $(\mathbf{u}, p)$ and $\left(\mathbf{u}_{h}, p_{h}\right)$ are the solutions of the continuous and of the modified discrete problems, and applying Green's theorem and Lemma 3.1 to get $\left(\nabla p, \Pi_{h} \mathbf{z}_{\mathbf{g}, h}\right)=0$, we end up with

$$
\begin{aligned}
\left(\mathbf{g}, \mathbf{u}-\mathbf{u}_{h}\right)= & \nu\left(\nabla_{h}\left(\mathbf{u}-\mathbf{u}_{h}\right), \nabla_{h}\left(\mathbf{z}_{\mathbf{g}}-\mathbf{z}_{\mathbf{g}, h}\right)\right)-\left(r_{\mathbf{g}}-r_{\mathbf{g}, h}, \operatorname{div}_{h}\left(\mathbf{u}-\mathbf{u}_{h}\right)\right) \\
& +\nu\left(\nabla \mathbf{u}, \nabla_{h}\left(\mathbf{z}_{\mathbf{g}, h}-\mathbf{z}_{\mathbf{g}}\right)\right)+\nu\left(\Delta \mathbf{u}, \Pi_{h}\left(\mathbf{z}_{\mathbf{g}, h}-\mathbf{z}_{\mathbf{g}}\right)\right)+\nu\left(\Delta \mathbf{u}, \Pi_{h} \mathbf{z}_{\mathbf{g}}-\mathbf{z}_{\mathbf{g}}\right) \\
& -\sum_{E \in \mathcal{E}_{h}}\left\{\left\langle\nu \frac{\partial \mathbf{z}_{\mathbf{g}}}{\partial \mathbf{n}_{E}},\left[\mathbf{u}-\mathbf{u}_{h}\right]_{E}\right\rangle_{E}-\left\langle r_{\mathbf{g}},\left[\mathbf{u}-\mathbf{u}_{h}\right]_{E} \cdot n_{E}\right\rangle_{E}\right\} \\
& -\sum_{E \subset \Gamma}\left\{\left\langle\nu \frac{\partial \mathbf{z}_{\mathbf{g}}}{\partial \mathbf{n}_{E}}, \mathbf{u}-\mathbf{u}_{h}\right\rangle_{E}-\left\langle r_{\mathbf{g}}, \mathbf{u}-\mathbf{u}_{h} \cdot \mathbf{n}_{E}\right\rangle_{E}\right\} .
\end{aligned}
$$

Now we estimate term by term. The Cauchy-Schwarz's inequality yields for the first and the second term in $(3.13)$

$$
\begin{aligned}
\nu \mid\left(\nabla_{h}\left(\mathbf{u}-\mathbf{u}_{h}\right), \nabla_{h}\left(\mathbf{z}_{\mathbf{g}}-\mathbf{z}_{\mathbf{g}, h}\right) \mid\right. & \leq C\left|\mathbf{u}-\mathbf{u}_{h}\right|_{1, h} h \nu\left|\mathbf{z}_{\mathbf{g}}\right|_{2} \leq C h\|\mathbf{g}\|_{0}\left|\mathbf{u}-\mathbf{u}_{h}\right|_{1, h}, \\
\left|\left(r_{\mathbf{g}}-r_{\mathbf{g}, h}, \operatorname{div}\left(\mathbf{u}-\mathbf{u}_{h}\right)\right)\right| & \leq C h\left|r_{\mathbf{g}}\right|_{1}\left|\mathbf{u}-\mathbf{u}_{h}\right|_{1, h} \leq C h\|\mathbf{g}\|_{0}\left|\mathbf{u}-\mathbf{u}_{h}\right|_{1, h}
\end{aligned}
$$

In order to estimate the third and fourth term in (3.13), we apply Lemma 3.2

$$
\nu \mid\left(\nabla \mathbf{u}, \nabla_{h}\left(\mathbf{z}_{\mathbf{g}, h}-\mathbf{z}_{\mathbf{g}}\right)\right)+\left(\Delta \mathbf{u},\left.\Pi_{h}\left(\mathbf{z}_{\mathbf{g}, h}-\mathbf{z}_{\mathbf{g}}\right)\left|\leq C \sum_{K \in \mathcal{T}_{h}} h_{K}^{k}\right| \mathbf{u}\right|_{k+1, K} \nu\left|\mathbf{z}_{\mathbf{g}, h}-\mathbf{z}_{\mathbf{g}}\right|_{1, K} \leq C h^{k+1}|\mathbf{u}|_{k+1}\|\mathbf{g}\|_{0} .\right.
$$


The operator $\Pi_{h}$ satisfies $(2.17)$, thus we can subtract the $\mathrm{L}^{2}$-projection $\pi_{k-2}^{K}: \mathrm{L}^{2}(K) \rightarrow P_{k-2}(K)$ and estimate the fifth term in (3.13) as follows

$$
\begin{aligned}
\nu\left|\left(\Delta \mathbf{u}, \Pi_{h} \mathbf{z}_{\mathbf{g}}-\mathbf{z}_{\mathbf{g}}\right)\right| & \leq \sum_{K \in \mathcal{T}_{h}} \nu\left|\left(\Delta \mathbf{u}-\pi_{k-2}^{K} \Delta \mathbf{u}, \Pi_{h} \mathbf{z}_{\mathbf{g}}-\mathbf{z}_{\mathbf{g}}\right)_{K}\right| \leq C \sum_{K \in \mathcal{T}_{h}} h_{K}^{k-1}|\mathbf{u}|_{k+1, K} h_{K}^{2} \nu\left|\mathbf{z}_{\mathbf{g}}\right|_{2, K} \\
& \leq C h^{k+1}|\mathbf{u}|_{k+1}\|\mathbf{g}\|_{0} .
\end{aligned}
$$

The two sums in (3.13) are estimated as in ([12], Lem. 3) using the $\mathrm{L}^{2}$-projection $\pi_{0}^{E}: \mathrm{L}^{2}(E) \rightarrow P_{0}(E)$. Since the ideas are the same, we show only the first of the two estimates

$$
\begin{aligned}
& \left|\sum_{E \in \mathcal{E}_{h}}\left\{\left\langle\nu \frac{\partial \mathbf{z}_{\mathbf{g}}}{\partial \mathbf{n}_{E}},\left[\mathbf{u}-\mathbf{u}_{h}\right]_{E}\right\rangle_{E}-\left\langle r_{\mathbf{g}},\left[\mathbf{u}-\mathbf{u}_{h}\right]_{E} \cdot \mathbf{n}_{E}\right\rangle_{E}\right\}\right|= \\
& \quad\left|\sum_{E \in \mathcal{E}_{h}}\left\{\left\langle\nu \frac{\partial \mathbf{z}_{\mathbf{g}}}{\partial \mathbf{n}_{E}}-\pi_{0}^{E} \frac{\partial \mathbf{z}_{\mathbf{g}}}{\partial \mathbf{n}_{E}},\left[\mathbf{u}-\mathbf{u}_{h}\right]_{E}\right\rangle_{E}-\left\langle r_{\mathbf{g}}-\pi_{0}^{E} r_{\mathbf{g}},\left[\mathbf{u}-\mathbf{u}_{h}\right]_{E} \cdot \mathbf{n}_{E}\right\rangle_{E}\right\}\right| \\
& \leq C \sum_{K \in T_{h}} h_{K}\left(\nu\left|\mathbf{z}_{\mathbf{g}}\right|_{2, K}+\left|r_{\mathbf{g}}\right|_{1, K}\right)\left|\mathbf{u}-\mathbf{u}_{h}\right|_{1, K} \leq C h\left|\mathbf{u}-\mathbf{u}_{h}\right|_{1, h}\|\mathbf{g}\|_{0} .
\end{aligned}
$$

Collecting all estimates, we obtain

$$
\left\|\mathbf{u}-\mathbf{u}_{h}\right\|_{0}=\sup _{\mathbf{g} \in \mathrm{L}^{2}(\Omega)^{d}} \frac{\left(\mathbf{g}, \mathbf{u}-\mathbf{u}_{h}\right)}{\|\mathbf{g}\|_{0}} \leq C\left(h^{k+1}|\mathbf{u}|_{k+1}+h\left|\mathbf{u}-\mathbf{u}_{h}\right|_{1, h}\right)
$$

from which the bound (3.12) follows.

Remark 3.6. Theorems 3.3 and 3.5 show that the velocity error in both the (broken) $\mathrm{H}^{1}$-semi-norm and the $\mathrm{L}^{2}$-norm is independent of the regularity of the pressure.

Remark 3.7. If $\mathbf{f}=\nabla \Phi$ with $\Phi \in \mathrm{H}^{1}(\Omega)$ then the solution $\mathbf{u}_{h}$ of the modified problem (2.20) satisfies

$$
\nu\left(\nabla_{h} \mathbf{u}_{h}, \nabla_{h} \mathbf{u}_{h}\right)=\left(\mathbf{f}, \Pi_{h} \mathbf{u}_{h}\right)=\left(\nabla \Phi, \Pi_{h} \mathbf{u}_{h}\right)=-\left(\Phi, \operatorname{div}_{h} \Pi_{h} \mathbf{u}_{h}\right)=0
$$

from which $\mathbf{u}_{h}=\mathbf{0}$ follows.

Remark 3.8. Velocity approximations $\mathbf{u}_{h} \neq \mathbf{0}$ of the solution $\mathbf{u}=\mathbf{0}$ of a continuous problem are often called spurious velocities [20,21]. Consider the case of a drop of liquid filling the domain $\Omega_{1}$ within an immiscible liquid separated by the interface $\Gamma_{F}$. We have to take into account the surface tension force which is distributed along the interface $\Gamma_{F}$. Introducing the velocity deformation tensor $\mathbb{D}(\mathbf{u})=\frac{1}{2}\left(\nabla \mathbf{u}+\nabla \mathbf{u}^{T}\right)$ and the sum of principal curvatures $\mathcal{K}$, a weak formulation of the equilibrium state reads:

Find $(\mathbf{u}, p) \in \mathbf{V} \times Q$ such that

$$
2 \nu(\mathbb{D}(\mathbf{u}), \mathbb{D}(\mathbf{v}))-(p, \operatorname{div} \mathbf{v})+(q, \operatorname{div} \mathbf{u})=\int_{\Gamma_{F}} \mathcal{K} \mathbf{v} \cdot \mathbf{n} \mathrm{d} \gamma \quad \text { for all }(\mathbf{v}, q) \in \mathbf{V} \times Q .
$$

Since $\Gamma_{F}$ is a sphere in the considered equilibrium, we have $\mathcal{K}=$ const. and conclude by setting $q=p$ and $\mathbf{v}=\mathbf{u}$ that

$$
2 \nu(\mathbb{D}(\mathbf{u}), \mathbb{D}(\mathbf{u}))=\mathcal{K} \int_{\Gamma_{F}} \mathbf{u} \cdot \mathbf{n} \mathrm{d} \gamma=\mathcal{K} \int_{\Omega_{1}} \operatorname{div} \mathbf{u} \mathrm{d} x=0 \quad \Rightarrow \quad \mathbf{u}=\mathbf{0}
$$

This property does not transfers to the discrete case and - in general (mesh unfitted to $\Omega_{1}$ ) - spurious velocities appear [20]. The modified scheme would read. 
Find $\left(\mathbf{u}_{h}, p_{h}\right) \in \mathbf{V}_{h} \times Q_{h}$ such that

$$
2 \nu\left(\mathbb{D}\left(\mathbf{u}_{h}\right), \mathbb{D}\left(\mathbf{v}_{h}\right)\right)-\left(p_{h}, \operatorname{div} \mathbf{v}_{h}\right)+\left(q_{h}, \operatorname{div} \mathbf{u}_{h}\right)=\int_{\Gamma_{F}} \mathcal{K} \Pi_{h} \mathbf{v}_{h} \cdot \mathbf{n} \mathrm{d} \gamma \quad \text { for all }\left(\mathbf{v}_{h}, q_{h}\right) \in \mathbf{V}_{h} \times Q_{h} .
$$

We obtain now by setting $q_{h}=p_{h}$ and $\mathbf{v}_{h}=\mathbf{u}_{h}$ that

$$
2 \nu\left(\mathbb{D}\left(\mathbf{u}_{h}\right), \mathbb{D}\left(\mathbf{u}_{h}\right)\right)=\mathcal{K} \int_{\Gamma_{F}} \Pi_{h} \mathbf{u}_{h} \cdot \mathbf{n} \mathrm{d} \gamma=\mathcal{K} \int_{\Omega_{1}} \operatorname{div} \Pi_{h} \mathbf{u}_{h} \mathrm{~d} x=0 \quad \Rightarrow \quad \mathbf{u}_{h}=\mathbf{0}
$$

even for meshes unfitted to $\Omega_{1}$ since the discretely divergence-free velocity field $\mathbf{u}_{h}$ is mapped to a divergence-free field.

\section{EXAMPLES}

We consider in this section triples of spaces $\left(\mathbf{V}_{h}, Q_{h}, \mathbf{X}_{h}\right)$ satisfying all assumptions made in the previous sections. Note that the pressure space is always fixed to be $Q_{h}=P_{k-1}^{\text {disc }}$.

\subsection{Families of simplicial meshes}

We consider for the space $\mathbf{X}_{h}$ the Raviart-Thomas spaces $\mathbf{R} \mathbf{T}_{k-1}, k \geq 2$. Let $K$ be a simplex in $\mathbb{R}^{d}$. Then, the local space of shape functions is given by

$$
\mathbf{R T}_{k-1}(K):=P_{k-1}(K)^{d}+\mathbf{x} P_{k-1}(K), \quad \operatorname{dim} \mathbf{R T}_{k-1}(K)= \begin{cases}k(k+2), & d=2 \\ k(k+1)(k+3) / 2, & d=3 .\end{cases}
$$

We get for $\mathbf{v}_{h} \in \mathbf{R T}_{k-1}(K)$ that $\operatorname{div} \mathbf{v}_{h} \in P_{k-1}(K)$. Taking into consideration that $\mathbf{x} \cdot \mathbf{n}_{E}$ is constant for $\mathbf{x} \in E$, we see that $\left.\mathbf{v}_{h} \cdot \mathbf{n}_{E}\right|_{E} \in P_{k-1}(E)$. Now we define $\mathbf{X}_{h}$ in the following way

$$
\mathbf{X}_{h}:=\left\{\mathbf{v}_{h} \in \mathbf{X}:\left.\mathbf{v}_{h}\right|_{K} \in \mathbf{R T}_{k-1}(K) \text { for all } K \in \mathcal{T}_{h},\left\langle q, \mathbf{v}_{h} \cdot \mathbf{n}\right\rangle_{E}=0 \text { for all } q \in P_{k-1}(E), E \subset \Gamma\right\} .
$$

Then, the properties (2.13)-(2.16) of Assumption A4 are satisfied by setting $s=k-1$.

\subsubsection{Conforming discretisations}

The space to approximate the velocity has to be rich enough to satisfy the inf-sup condition A1. This can be achieved by enriching the space $P_{k}$ of continuous, piece-wise polynomial functions of degree less than or equal to $k$ with suitable bubble functions. Let $b_{K}$ be the product of barycentric coordinates related to the simplex $K \subset \mathbb{R}^{d}, d=2,3$, and let $\widetilde{P}_{l}(K)$ denote the space of homogeneous polynomials of degree $l$ which means $\widetilde{P}_{l}(K)=\operatorname{span}\left\{x_{1}^{i} x_{2}^{l-i}, 0 \leq i \leq l\right\}$ for $d=2$ and $\widetilde{P}_{l}(K)=\operatorname{span}\left\{x_{1}^{i_{1}} x_{2}^{i_{2}} x_{3}^{l-i_{1}-i_{2}}, 0 \leq i_{1}+i_{2} \leq l\right\}$ for $d=3$, respectively. We choose

$$
\mathbf{V}_{h}:=\left\{\mathbf{v}_{h} \in \mathrm{H}_{0}^{1}(\Omega)^{2}:\left.\mathbf{v}_{h}\right|_{K} \in P_{k}^{+}(K)^{2}\right\} \quad \text { with } \quad P_{k}^{+}(K):=P_{k}(K) \oplus \operatorname{span}\left\{b_{K} \widetilde{P}_{k-2}(K)\right\}, \quad k \geq 2,
$$

in the two-dimensional case whereas we set

$\mathbf{V}_{h}:=\left\{\mathbf{v}_{h} \in \mathrm{H}_{0}^{1}(\Omega)^{3}:\left.\mathbf{v}_{h}\right|_{K} \in P_{k}^{+}(K)^{3}\right\} \quad$ with $\quad P_{k}^{+}(K):=P_{k}(K) \oplus \operatorname{span}\left\{b_{K}\left(\widetilde{P}_{k-2}(K) \oplus \widetilde{P}_{k-3}(K)\right)\right\}, \quad k \geq 3$,

in the three-dimensional case. The enrichment in the case $d=3$ and $k=2$ has to be handled separately ([22], Chap. II, Sect. 2.3). We choose the vector-valued enrichment space

$$
\mathbf{P}_{2}^{+}(K):=\left(P_{2}(K) \oplus b_{K} P_{0}(K)\right)^{3} \oplus \operatorname{span}\left\{\mathbf{p}_{1}, \mathbf{p}_{2}, \mathbf{p}_{3}, \mathbf{p}_{4}\right\}
$$


with the face bubble functions $\mathbf{p}_{i}=\mathbf{n}_{i} \lambda_{i+1} \lambda_{i+2} \lambda_{i+3}, i=1,2,3,4$, where $\mathbf{n}_{i}$ is the outer normal of the face opposite to the vertex $\lambda_{i}=1$ and all indices are modulo 4 . The finite element space will be given by

$$
\mathbf{V}_{h}:=\left\{\mathbf{v}_{h} \in \mathrm{H}_{0}^{1}(\Omega)^{3}:\left.\mathbf{v}_{h}\right|_{K} \in \mathbf{P}_{2}^{+}(K)\right\}
$$

in the considered special case.

All above given finite element spaces for velocity approximations combined with the pressure space $Q_{h}=P_{k-1}^{\text {disc }}$ satisfy the inf-sup condition ([22], Chap. II, Sects. 2.2 and 2.3), thus A1 is satisfied. A2 holds for any $r \geq 0$ due to the continuity of the velocity space. The standard interpolant $i_{h}: \mathbf{V} \cap \mathrm{H}^{k+1}(\Omega)^{d} \rightarrow \mathbf{V}_{h}$ satisfies A3. It remains to show the existence of the reconstruction operator $\Pi_{h}: \mathbf{V} \rightarrow \mathbf{X}_{h}$ satisfying (2.17)-(2.19). The first two relations, (2.17) and (2.18), are used to define the reconstruction operator $\Pi_{h}$ locally which is well defined ([3], Prop. 2.3.4). Then, setting $\left.\left(\Pi_{h} \mathbf{v}\right)\right|_{K}=\left.\Pi_{h}(\mathbf{v})\right|_{K}$ we see that this construction guarantees the continuity of the normal component $\mathbf{v}_{h} \cdot \mathbf{n}_{E}$ across an element, thus $\Pi_{h} \mathbf{v} \in \mathbf{X}_{h}$. The local approximation property (2.19) follows from $\Pi_{h} \mathbf{v}=\mathbf{v}$ for all $\mathbf{v} \in P_{k-1}(K), k \geq 2$, and the Bramble-Hilbert's Lemma [5] applied on the reference cell.

Conclusion 4.1. Theorems 3.3 and 3.5 hold for the triple $\left(\mathbf{V}_{h}, Q_{h}, \mathbf{X}_{h}\right)=\left(\mathbf{P}_{k}^{+}, P_{k-1}^{\text {disc }}, \mathbf{R T}_{k-1}\right), k \geq 2$.

Note that the operator from ([35], Sect. 4.2) could also used in this case.

\subsubsection{Nonconforming discretisations}

Nonconforming finite element approximations on triangles of order $k=1$ and $k=3$ satisfying the inf-sup condition have been already proposed in [12]. Here, we consider a family of elements of arbitrary order in the two-dimensional case [34]. We choose

$$
\begin{array}{r}
\mathbf{V}_{h}:=\left\{\mathbf{v}_{h} \in \mathrm{L}^{2}(\Omega)^{2}:\left.\mathbf{v}_{h}\right|_{K} \in P_{k}^{\mathrm{nc}}(K)^{2},\right. \\
\left\langle q,\left[\mathbf{v}_{h}\right]\right\rangle_{E}=\mathbf{0} \text { for all } q \in P_{k-1}(E), E \in \mathcal{E}_{h}, \\
\left.\left\langle q, \mathbf{v}_{h}\right\rangle_{E}=\mathbf{0} \text { for all } q \in P_{k-1}(E), E \subset \Gamma\right\}
\end{array}
$$

where the local space of shape function is given by

$$
P_{k}^{\mathrm{nc}}(K):=P_{k}(K)+\operatorname{span}\left\{b_{K}^{*} \lambda_{1}^{k-2-i} \lambda_{2}^{i}: i=0, \ldots, k-2\right\}, \quad b_{K}^{*}=\left(\lambda_{1}-\lambda_{2}\right)\left(\lambda_{2}-\lambda_{3}\right)\left(\lambda_{3}-\lambda_{1}\right),
$$

and $\lambda_{1}, \lambda_{2}, \lambda_{3}$ are the barycentric coordinates with respect to $K$. Note that the enrichment depends on the choice which edge $E$ corresponds to $\lambda_{3}=0$. However, the enriched space $P_{k}^{\text {nc }}(K)$ is independent of that choice ([34], Sect. 4). The finite element pair $\left(P_{k}^{\text {nc }}, P_{k-1}^{\text {disc }}\right), k \geq 1$, satisfies the inf-sup condition ([34], Thm. 2), thus A1 holds. We mention that the lowest order pair $(k=1)$ of this family is the Crouzeix-Raviart element for which a reconstruction operator has been studied in [31]. A2 is satisfied with $r=k-1$ by the definition of $\mathbf{V}_{h}$. Choosing the same nodal functionals as in [34], the existence of an interpolation $i_{h}: \mathbf{V} \cap \mathrm{H}^{k+1}(\Omega)^{2} \rightarrow \mathbf{V}_{h}$ satisfying (2.7) follows ([34], Lem. 4), i.e., A3 holds. Above we already proved the properties (2.13)-(2.16) of Assumption A4 for $s=k-1$. It remains to show the existence of the reconstruction operator $\Pi_{h}: \mathbf{V}+\mathbf{V}_{h} \rightarrow \mathbf{X}_{h}$ satisfying (2.17)(2.19). This can be done as in the conforming case by using (2.17) and (2.18) to define $\Pi_{h}$ locally on each cell. Again, the approximation property (2.19) follows from the Bramble-Hilbert's Lemma applied on the reference triangle.

Conclusion 4.2. Theorems 3.3 and 3.5 hold for the triple $\left(\mathbf{V}_{h}, Q_{h}, \mathbf{X}_{h}\right)=\left(\mathbf{P}_{k}^{\text {nc }}, P_{k-1}^{\text {disc }}, \mathbf{R} \mathbf{T}_{k-1}\right), k \geq 2$.

Remark 4.3. A third order nonconforming finite element pair different from the one considered here has been proposed in ([12], Exp. 5). The space for approximating the velocity is given by

$$
\begin{array}{r}
\mathbf{V}_{h}:=\left\{\mathbf{v}_{h} \in \mathrm{L}^{2}(\Omega)^{2}:\left.\mathbf{v}_{h}\right|_{K} \in \widehat{P}_{3}^{\mathrm{nc}}(K)^{2},\right. \\
,\left\langle q,\left[\mathbf{v}_{h}\right]\right\rangle_{E}=\mathbf{0} \text { for all } q \in P_{2}(E), E \in \mathcal{E}_{h}, \\
\left.\left\langle q, \mathbf{v}_{h}\right\rangle_{E}=\mathbf{0} \text { for all } q \in P_{2}(E), E \subset \Gamma\right\}
\end{array}
$$


where the local space of shape functions is defined as

$$
\widehat{P}_{3}^{\mathrm{nc}}(K):=P_{3}(K)+\operatorname{span}\left\{\left(\lambda_{1} \lambda_{2} \lambda_{3}\right) P_{1}(K)\right\} .
$$

The pair $\left(\widehat{P}_{3}^{\text {nc }}, P_{2}^{\text {disc }}\right)$ satisfies the inf-sup condition A1. Assumption A2 holds for $r=2$ due to the definition of $\mathbf{V}_{h}$. We can define locally an interpolation $i_{h}: \mathbf{V} \cap \mathrm{H}^{k+1}(\Omega)^{d} \rightarrow \mathbf{V}_{h}$ which satisfies $A 3$ (see [12], Lem. 5). A4 can be shown as before. Thus, setting $k=3$, Theorems 3.3 and 3.5 hold for the triple $\left(\mathbf{V}_{h}, Q_{h}, \mathbf{X}_{h}\right)=$ $\left(\widehat{\mathbf{P}}_{3}^{\text {nc }}, P_{2}^{\text {disc }}, \mathbf{R T}_{2}\right)$.

\subsection{Families of rectangular and brick meshes}

We now consider rectangular $(d=2)$ and brick $(d=3)$ meshes. We choose for the space $\mathbf{X}_{h}$ the BrezziDouglas-Marini's spaces $\mathbf{B D M}_{k}, k \geq 2$ (see [3], Sect. 2.4). We start with the two-dimensional case and set

$$
\mathbf{B D M}_{k}(K):=P_{k}(K)^{2}+\operatorname{span}\left\{\left(\begin{array}{c}
-x_{1}^{k+1} \\
(k+1) x_{1}^{k} x_{2}
\end{array}\right),\left(\begin{array}{c}
(k+1) x_{1} x_{2}^{k} \\
-x_{2}^{k+1}
\end{array}\right)\right\},
$$

$\operatorname{dim} \mathbf{B D M}_{k}=k^{2}+3 k+4$. Taking into consideration that the standard vector-valued $P_{k}(K)^{2}$ space is locally enriched by two divergence-free functions, we conclude that $\operatorname{div} \mathbf{v}_{h} \in P_{k-1}(K)$ for $\mathbf{v}_{h} \in \mathbf{B D M}_{k}(K)$. Furthermore, $\left.\mathbf{v}_{h} \cdot \mathbf{n}_{E}\right|_{E} \in P_{k}(E)$ for $\mathbf{v}_{h} \in \mathbf{B D M}_{k}(K)$. We are ready to define $\mathbf{X}_{h}$ as

$$
\mathbf{X}_{h}:=\left\{\mathbf{v}_{h} \in \mathbf{X}:\left.\mathbf{v}_{h}\right|_{K} \in \mathbf{B D M}_{k}(K) \text { for all } K \in \mathcal{T}_{h},\left\langle q, \mathbf{v}_{h} \cdot \mathbf{n}\right\rangle_{E}=0 \text { for all } q \in P_{k}(E), E \subset \Gamma\right\} .
$$

Note that $\mathbf{v}_{h} \in \mathbf{X}$ implies that the normal component $\mathbf{v}_{h} \cdot \mathbf{n}_{E}$ is continuous across edges $E \in \mathcal{E}_{h}$. Then, the properties (2.13)-(2.16) of Assumption A4 are satisfied by setting $s=k$.

We turn over to the three-dimensional case. According to [2], we set

$$
\mathbf{B D M}_{k}(K):=P_{k}(K)^{3}+\operatorname{span}\left\{\operatorname{curl}\left(\begin{array}{l}
x_{2} x_{3}\left(w_{2}\left(x_{1}, x_{3}\right)-w_{3}\left(x_{1}, x_{2}\right)\right) \\
x_{3} x_{1}\left(w_{3}\left(x_{1}, x_{2}\right)-w_{1}\left(x_{2}, x_{3}\right)\right) \\
x_{1} x_{2}\left(w_{1}\left(x_{2}, x_{3}\right)-w_{2}\left(x_{1}, x_{3}\right)\right)
\end{array}\right)\right\},
$$

$\operatorname{dim} \mathbf{B D M}_{k}(K)=(k+1)\left(k^{2}+5 k+12\right) / 2$. Again the space $P_{k}(K)^{3}$ is locally enriched by divergence-free functions where $w_{1}, w_{2}$, and $w_{3}$ belong to $\widetilde{P}_{k}$. As a consequence, we have div $\mathbf{v}_{h} \in P_{k-1}(K)$ for $\mathbf{v}_{h} \in \mathbf{B D M}_{k}(K)$. Furthermore, a short computation shows that $\left.\mathbf{v}_{h} \cdot \mathbf{n}_{E}\right|_{E} \in P_{k}(E)$ for $\mathbf{v}_{h} \in \mathbf{B D M}_{k}(K)$. We define

$$
\mathbf{X}_{h}:=\left\{\mathbf{v}_{h} \in \mathbf{X}:\left.\mathbf{v}_{h}\right|_{K} \in \mathbf{B D M}_{k}(K) \text { for all } K \in \mathcal{T}_{h},\left\langle q, \mathbf{v}_{h} \cdot \mathbf{n}\right\rangle_{E}=0 \text { for all } q \in P_{k}(E), E \subset \Gamma\right\}
$$

in the three-dimensional case. Then, the properties (2.13)-(2.16) of Assumption A4 are satisfied by setting $s=k$.

\subsubsection{Conforming discretisations}

Consider velocity approximations in the space

$$
\mathbf{V}_{h}:=\left\{\mathbf{v}_{h} \in \mathrm{H}_{0}^{1}(\Omega)^{d}:\left.\mathbf{v}_{h}\right|_{K} \in Q_{k}(K)^{d}\right\} .
$$

Then, the pair of finite elements $\left(\mathbf{V}_{h}, Q_{h}\right)=\left(\mathbf{Q}_{k}, P_{k-1}^{\text {disc }}\right)$ is inf-sup stable in any space dimensions [33], in particular for $d=2$ and $d=3$, thus A1 holds. A2 is true for any $r \geq 0$ since the functions $\mathbf{v}_{h} \in \mathbf{V}_{h}$ are globally continuous. The usual Lagrange interpolation $i_{h}: \mathbf{V} \cap \mathrm{H}^{k+1}(\Omega)^{d} \rightarrow \mathbf{V}_{h}$ satisfies A3. Now we show the remaining conditions of Assumption A4. The two relations (2.17) and (2.18) define the reconstruction operator $\Pi_{h}$ locally (see [3], Prop. 2.4.2) and ([2], Thm. 3.6). Then, setting $\left.\left(\Pi_{h} \mathbf{v}\right)\right|_{K}=\left.\Pi_{h}(\mathbf{v})\right|_{K}$ we see that this construction guarantees the continuity of the normal component $\mathbf{v}_{h} \cdot \mathbf{n}_{E}$ across an edge/face, thus $\Pi_{h} \mathbf{v} \in \mathbf{X}_{h}$. The local approximation property (2.19) follows from $\Pi_{h} \mathbf{v}=\mathbf{v}$ for all $\mathbf{v} \in P_{k-1}(K), k \geq 2$, and the Bramble-Hilbert's Lemma applied on the reference cell.

Conclusion 4.4. Theorems 3.3 and 3.5 hold for the triple $\left(\mathbf{V}_{h}, Q_{h}, \mathbf{X}_{h}\right)=\left(\mathbf{Q}_{k}, P_{k-1}^{\text {disc }}, \mathbf{B D} \mathbf{M}_{k}\right), k \geq 2$. 


\subsubsection{Nonconforming discretisations}

Several families of inf-sup stable nonconforming finite element pairs on quadrilaterals and hexahedra are given already in literature, see $[1,32]$. However, they are not suitable for defining velocity reconstruction operators into BDM-spaces due to limited consistency across the elements sides.

We construct the nonconforming finite element spaces of the velocity approximation by

$$
\begin{array}{r}
\mathbf{V}_{h}:=\left\{\mathbf{v}_{h} \in \mathrm{L}^{2}(\Omega)^{d}:\left.\mathbf{v}_{h}\right|_{K} \in Q_{k}^{\mathrm{nc}}(K)^{d},\left\langle q,\left[\mathbf{v}_{h}\right]\right\rangle_{E}=\mathbf{0}, \text { for all } q \in P_{k}(E), E \in \mathcal{E}_{h},\right. \\
\left.\left\langle q, \mathbf{v}_{h}\right\rangle_{E}=\mathbf{0}, \text { for all } q \in P_{k}(E), E \subset \Gamma\right\}
\end{array}
$$

where the local function space $Q_{k}^{\text {nc }}$ are defined via

$$
Q_{k}^{\mathrm{nc}}(K):=\left\{v: v \circ F_{K} \in \widehat{Q}_{k}^{\mathrm{nc}}\right\}
$$

with the affine reference transformation $F_{K}:(-1,1)^{d} \rightarrow K$. In order to complete the definition of $\mathbf{V}_{h}$, the spaces $\widehat{Q}_{k}^{\text {nc }}$ on the reference cells $(-1,1)^{d}$ have to be specified. Assumption A2 is fulfilled for $r=k$ due to the definition of $\mathbf{V}_{h}$.

We start with the two-dimensional case. Using the cell moments which correspond to $P_{k-2}(\widehat{K})$ and the edge moments which are associated to $P_{k}\left(\widehat{E}_{i}\right), i=1,2,3,4$, Algorithm 1 in [25] allows to construct the local function space

$$
\widehat{Q}_{k}^{\mathrm{nc}}:=P_{k}(\widehat{K}) \oplus \widehat{R}_{k} \oplus \widehat{S}_{k}
$$

with

$$
\widehat{S}_{k}:=\operatorname{span}\left\{L_{i i}, L_{i+1, i}, L_{i, i+1}, L_{i+2, i}-L_{i, i+2}: k / 2<i \leq k\right\}
$$

and

$$
\widehat{R}_{k}:= \begin{cases}\operatorname{span}\left\{L_{i+1, i}, L_{i, i+1}, L_{i+2, i}-L_{i, i+2},: i=k / 2\right\}, & k \text { even, } \\ \operatorname{span}\left\{L_{i+2, i}-L_{i, i+2}: i=(k-1) / 2\right\}, & k \text { odd },\end{cases}
$$

where $L_{\alpha \beta}(\xi, \eta):=L_{\alpha}(\xi) L_{\beta}(\eta)$ are polynomials in two variables. According to ([25], Lem. 1), the interpolation operator based on the cell and edge moments is well defined. Furthermore, the inclusion $P_{k}(\widehat{K}) \subset \widehat{Q}_{k}^{\text {nc }}$ ensures the approximation properties of Assumption A3.

We now pass to the three-dimensional case. Algorithm 2 in [25] with cell moments associated to $P_{k-2}(\widehat{K})$ and face moments which correspond to $P_{k}\left(\widehat{F}_{i}\right), i=1, \ldots, 6$, provides the function space

$$
\widehat{Q}_{k}^{\mathrm{nc}}:=P_{k-2}(\widehat{K}) \oplus \bigoplus_{i=1}^{3} \widehat{A}_{i} \oplus \bigoplus_{i=1}^{3} \widehat{B}_{i} \oplus \widehat{C}
$$

where

$$
\begin{aligned}
& \widehat{A}_{1}:=\operatorname{span}\left\{L_{i, j, m}, L_{i+1, j, m}: i>\max (j, k), i+j+m=k\right\}, \\
& \widehat{A}_{2}:=\operatorname{span}\left\{L_{i, j, m}, L_{i, j+1, m}: j>\max (i, k), i+j+m=k\right\}, \\
& \widehat{A}_{3}:=\operatorname{span}\left\{L_{i, j, m}, L_{i, j, m+1}: m>\max (i, j), i+j+m=k\right\},
\end{aligned}
$$


and

$$
\begin{aligned}
& \widehat{B}_{1}:=\operatorname{span}\left\{L_{j i i}, L_{j, i+1, i}, L_{j, i, i+1}, L_{j, i, i+2}-L_{j, i+2, i}: i>j, k \leq 2 i+j \leq 2 k-j\right\}, \\
& \widehat{B}_{2}:=\operatorname{span}\left\{L_{i j i}, L_{i+1, j, i}, L_{i, j, i+1}, L_{i, j, i+2}-L_{i+2, j, i}: i>j, k \leq 2 i+j \leq 2 k-j\right\}, \\
& \widehat{B}_{3}:=\operatorname{span}\left\{L_{i i j}, L_{i+1, i, j}, L_{i, i+1, j}, L_{i, i+2, j}-L_{i+2, i, j}: i>j, k \leq 2 i+j \leq 2 k-j\right\} .
\end{aligned}
$$

Furthermore, we set

$$
\begin{aligned}
\widehat{C}:= & \operatorname{span}\left\{L_{i i i}, L_{i+1, i, i}, L_{i, i+1, i}, L_{i, i, i+1},: k / 3 \leq i \leq k / 2\right\} \\
& \oplus \operatorname{span}\left\{L_{i+2, i, i}-L_{i, i+2, i}, L_{i, i+2, i}-L_{i, i, i+2},: k / 3 \leq i \leq k / 2\right\}
\end{aligned}
$$

where $L_{\alpha \beta \gamma}(\xi, \eta, \zeta):=L_{\alpha}(\xi) L_{\beta}(\eta) L_{\gamma}(\zeta)$ is a polynomial in three variables. Lemma 2 of [25] guarantees that the interpolation operator $i_{h}$ based on the cell and faces moments is well defined. Using the same ideas as in Example 10 in [25], the inclusion $P_{k}(\widehat{K}) \subset \widehat{Q}_{k}^{\text {nc }}$ can be shown. Hence, the approximation properties of Assumption A3 are provided.

For both considered space dimensions, the reconstruction operator $\Pi_{h}$ can be defined by using (2.17) and (2.18). Applying the Bramble-Hilbert's lemma shows that the approximation property (2.20) is satisfied. Hence, Assumption A4 is fulfilled.

It remains to show that the nonconforming velocity space $\mathbf{V}_{h}$ together with $Q_{h}=P_{k-1}^{\text {disc }}$ fulfils the inf-sup condition of Assumption A1. To this end, we use the equivalence of the inf-sup condition to the existence of an interpolation operator $I_{h}$ with

$$
\begin{array}{ccrl}
\left(\operatorname{div}_{h} I_{h} \mathbf{v}, q_{h}\right) & =\left(\operatorname{div} \mathbf{v}, q_{h}\right) & & \forall q_{h} \in Q_{h}, \mathbf{v} \in \mathrm{H}_{0}^{1}(\Omega)^{d}, \\
\left|I_{h} \mathbf{v}\right|_{1, h} \leq C|\mathbf{v}|_{1}, & & \forall \mathbf{v} \in \mathrm{H}_{0}^{1}(\Omega)^{d},
\end{array}
$$

where the constant $C$ is independent of $h$. This result is due to Fortin [17]. We will show that the interpolation operator $i_{h}$ based on cell and edge/face moments provides the properties (4.1) and (4.2). To check (4.1), we have

$$
\begin{aligned}
\left(\operatorname{div}_{h} i_{h} \mathbf{v}, q_{h}\right) & =\sum_{K \in \mathcal{T}_{h}}\left(\operatorname{div}_{h} i_{h}^{K} \mathbf{v}, q_{h}\right)_{K} \\
& =\sum_{K \in \mathcal{T}_{h}}\left(-\left(i_{h}^{K} \mathbf{v}, \nabla q_{h}\right)_{K}+\sum_{E \subset \partial K}\left\langle i_{r}^{K} \mathbf{v} \cdot \mathbf{n}_{K}, q_{h}\right\rangle_{E}\right) \\
& =\sum_{K \in \mathcal{T}_{h}}\left(-\left(\mathbf{v}, \nabla q_{h}\right)_{K}+\sum_{E \subset \partial K}\left\langle\mathbf{v} \cdot \mathbf{n}_{K}, q_{h}\right\rangle_{E}\right) \\
& =\left(\operatorname{div} \mathbf{v}, q_{h}\right) .
\end{aligned}
$$

We have used here an integration by parts and the fact that the restriction of $q_{h} \in Q_{h}$ to any edge/face $E$ belongs to $P_{k}(E)$ and that $\left.\nabla q_{h}\right|_{K} \in\left(P_{r-2}(K)\right)^{d}$. Moreover, the interpolation properties were exploited. The condition (4.2) follows from a generalisation of (2.7) to functions of $\mathrm{H}_{0}^{1}(\Omega)^{d}$.

Conclusion 4.5. Theorems 3.3 and 3.5 are satisfied for the triple $\left(\mathbf{V}_{h}, Q_{h}, \mathbf{X}_{h}\right)=\left(\mathbf{Q}_{k}^{\text {nc }}, P_{k-1}^{\text {disc }}, \mathbf{B D M}_{k}\right), k \geq 2$.

\section{NumericAl TESTS}

We will present in this section selected results of our numerical tests on the unit square $(0,1)^{2}$ using conforming and nonconforming discretisations of orders two to five on axiparallel rectangular cells. All calculations have been carried out using Matlab. 
TABLE 1. No flow problem, $\nu=1$, conforming elements of order 3.

\begin{tabular}{|c|c|c|c|c|c|c|c|c|c|c|c|c|}
\hline \multirow[b]{3}{*}{ Mesh } & \multicolumn{7}{|c|}{ Standard scheme } & \multicolumn{5}{|c|}{ Modified scheme } \\
\hline & \multicolumn{2}{|c|}{$\left\|\mathbf{u}-\mathbf{u}_{h}\right\|_{0}$} & \multicolumn{2}{|c|}{$\left|\mathbf{u}-\mathbf{u}_{h}\right|_{1}$} & \multicolumn{2}{|c|}{$\left\|p-p_{h}\right\|_{0}$} & \multirow{2}{*}{$\begin{array}{c}\left\|p_{h}-j_{h} p\right\|_{0} \\
\text { error }\end{array}$} & \multirow{2}{*}{$\begin{array}{c}\left\|\mathbf{u}-\mathbf{u}_{h}\right\|_{0} \\
\text { error } \\
\end{array}$} & \multirow{2}{*}{$\begin{array}{c}\mathbf{u}-\left.\mathbf{u}_{h}\right|_{1} \\
\text { error }\end{array}$} & \multicolumn{2}{|c|}{$\left\|p-p_{h}\right\|_{0}$} & \multirow{2}{*}{$\begin{array}{c}\left\|p_{h}-j_{h} p\right\|_{0} \\
\text { error }\end{array}$} \\
\hline & error & ord & error & ord & error & ord & & & & error & ord & \\
\hline $2 \times 3$ & $5.192-05$ & & $1.166-03$ & & $2.265-03$ & & $9.237-04$ & $1.687-18$ & $2.390-17$ & $2.068-03$ & & $5.661-17$ \\
\hline $4 \times$ & $3.966-06$ & 3.71 & $1.910-04$ & 2.61 & $3.095-04$ & 2.87 & 1.079-04 & $2.085-18$ & $2.774-17$ & 2.901-04 & 2.83 & $6.446-17$ \\
\hline $8 \times 12$ & $2.791-07$ & 3.83 & $2.788-05$ & 2.78 & $3.881-05$ & 3.00 & $1.083-05$ & $3.905-18$ & $4.310-17$ & $3.727-05$ & 2.96 & $1.478-16$ \\
\hline $16 \times 24$ & $1.853-08$ & 3.91 & $3.791-06$ & 2.88 & $4.800-06$ & 3.02 & $1.019-06$ & $7.737-18$ & $8.051-17$ & $4.690-06$ & 2.99 & $3.073-16$ \\
\hline $32 \times 48$ & $1.193-09$ & 3.96 & $4.950-07$ & 2.94 & $5.946-07$ & 3.01 & $9.283-08$ & $1.553-17$ & $1.591-16$ & $5.873-07$ & 3.00 & $6.245-16$ \\
\hline $64 \times 96$ & 7.563-11 & 3.98 & $6.327-08$ & 2.97 & 7.391-08 & 3.01 & 8.331-09 & $3.112-17$ & $3.177-16$ & $7.344-08$ & 3.00 & $1.260-15$ \\
\hline
\end{tabular}

TABLE 2. No flow problem, $\nu=1$, nonconforming elements of order 3 .

\begin{tabular}{|c|c|c|c|c|c|c|c|c|c|c|c|c|}
\hline \multirow[b]{3}{*}{ Mesh } & \multicolumn{7}{|c|}{ Standard scheme } & \multicolumn{5}{|c|}{ Modified scheme } \\
\hline & \multicolumn{2}{|c|}{$\left\|\mathbf{u}-\mathbf{u}_{h}\right\|_{0}$} & \multicolumn{2}{|c|}{$\left|\mathbf{u}-\mathbf{u}_{h}\right|_{1, h}$} & \multicolumn{2}{|c|}{$\left\|p-p_{h}\right\|_{0}$} & \multirow{2}{*}{$\begin{array}{c}\left\|p_{h}-j_{h} p\right\|_{0} \\
\text { error }\end{array}$} & \multirow{2}{*}{$\begin{array}{c}\left\|\mathbf{u}-\mathbf{u}_{h}\right\|_{0} \\
\text { error }\end{array}$} & \multirow{2}{*}{$\begin{array}{c}\left|\mathbf{u}-\mathbf{u}_{h}\right|_{1, h} \\
\text { error }\end{array}$} & \multicolumn{2}{|c|}{$\left\|p-p_{h}\right\|_{0}$} & \multirow{2}{*}{$\begin{array}{c}\left\|p_{h}-j_{h} p\right\|_{0} \\
\text { error }\end{array}$} \\
\hline & error & ord & error & ord & error & ord & & & & error & ord & \\
\hline $2 \times$ & $3.390-05$ & & $7.998-04$ & & $2.244-03$ & & $8.691-04$ & $5.222-19$ & $7.243-18$ & $2.068-03$ & & $3.060-17$ \\
\hline $4 \times$ & $2.923-06$ & 3.54 & $1.603-04$ & 2.32 & $3.057-04$ & 2.88 & $9.641-05$ & $6.668-19$ & $7.484-18$ & 2.901-04 & 2.83 & $2.907-17$ \\
\hline $8 \times 12$ & $2.137-07$ & 3.77 & $2.469-05$ & 2.70 & $3.837-05$ & 2.99 & $9.114-06$ & $1.202-18$ & $1.056-17$ & $3.727-05$ & 2.96 & $3.408-17$ \\
\hline $16 \times 24$ & $1.432-08$ & 3.90 & $3.397-06$ & 2.86 & $4.762-06$ & 3.01 & $8.243-07$ & $2.125-18$ & $1.754-17$ & 4.690-06 & 2.99 & $5.093-17$ \\
\hline $32 \times 48$ & $9.240-10$ & 3.95 & 4.444-07 & 2.93 & $5.919-07$ & 3.01 & $7.350-08$ & $3.978-18$ & $3.248-17$ & 5.873-07 & 3.00 & $9.026-17$ \\
\hline $64 \times 96$ & $5.863-11$ & 3.98 & $5.682-08$ & 2.97 & 7.373-08 & 3.00 & $6.521-09$ & 7.974-18 & $6.518-17$ & 7.344-08 & 3.00 & $1.791-16$ \\
\hline
\end{tabular}

\subsection{No flow problem}

We consider the classical Stokes problem $(\nu=1)$ with meshes of $n \times m$ rectangles. The right-hand side of the Stokes problem is given by the gradient force

$$
\mathbf{f}=\nabla \varphi, \quad \varphi=2 x^{2}(1-x) y(1-y)
$$

Hence, the solution of the Stokes problem is given by

$$
\mathbf{u}=\mathbf{0}, \quad p=\varphi-\frac{1}{36}
$$

Note that subtracting the constant ensures $p \in \mathrm{L}_{0}^{2}(\Omega)$.

Tables 1 and 2 present the errors for the standard and modified schemes applied to conforming and nonconforming discretisations using elements of third order. It can be seen that the standard scheme provides for the velocity approximations with both conforming and nonconforming finite elements the optimal convergence order of 3 for the (broken) $\mathrm{H}^{1}$-semi-norm of the velocity and the $\mathrm{L}^{2}$-norm of the pressure while the $\mathrm{L}^{2}$-norm of the velocity convergences with order 4 . However, the standard scheme violates the fundamental invariance condition given Remark 3.7 since the applied gradient force on the right-hand side induces a velocity field which does not vanish. In contrast, the modified scheme generates for both conforming and nonconforming discretisations the expected zero flow field. In addition, we observe that the modification influences the $\mathrm{L}^{2}$-error of the pressure only slightly. Furthermore, the discrete pressure $p_{h}$ of the modified discrete scheme coincides for the no flow problem with the $\mathrm{L}^{2}$-projection $j_{h} p$ of the continuous pressure $p$, as predicted by (3.6) of Theorem 3.3. This is not the case for the standard scheme. 
TABle 3. Problem with flow, $\nu=1$, conforming elements of order 4 .

\begin{tabular}{|c|c|c|c|c|c|c|c|c|c|c|c|c|}
\hline \multirow[b]{3}{*}{ Mesh } & \multicolumn{6}{|c|}{ Standard scheme } & \multicolumn{6}{|c|}{ Modified scheme } \\
\hline & \multicolumn{2}{|c|}{$\left\|\mathbf{u}-\mathbf{u}_{h}\right\|_{0}$} & \multicolumn{2}{|c|}{$\left|\mathbf{u}-\mathbf{u}_{h}\right|_{1}$} & \multicolumn{2}{|c|}{$\left\|p-p_{h}\right\|_{0}$} & \multicolumn{2}{|c|}{$\left\|\mathbf{u}-\mathbf{u}_{h}\right\|_{0}$} & \multicolumn{2}{|c|}{$\left|\mathbf{u}-\mathbf{u}_{h}\right|_{1}$} & \multicolumn{2}{|c|}{$\left\|p-p_{h}\right\|_{0}$} \\
\hline & error & ord & error & ord & error & ord & error & ord & error & ord & error & ord \\
\hline $2 \times 3$ & $1.075-05$ & & $2.863-04$ & & $4.114-04$ & & $4.613-05$ & & $1.217-03$ & & $8.830-04$ & \\
\hline $4 \times$ & $3.902-07$ & 4.78 & $2.033-05$ & 3.82 & $2.637-05$ & 3.96 & $2.017-06$ & 4.52 & $1.012-04$ & 3.59 & $5.811-05$ & 3.93 \\
\hline $8 \times 12$ & $1.308-08$ & 4.90 & $1.352-06$ & 3.91 & $1.632-06$ & 4.01 & $7.227-08$ & 4.80 & 7.074-06 & 3.84 & $3.225-06$ & 4.17 \\
\hline $16 \times 24$ & $4.224-10$ & 4.95 & $8.723-08$ & 3.95 & $1.010-07$ & 4.01 & 2.401-09 & 4.91 & $4.646-07$ & 3.93 & $1.789-07$ & 4.17 \\
\hline $32 \times 48$ & $1.341-11$ & 4.98 & $5.539-09$ & 3.98 & $6.279-09$ & 4.01 & $7.724-11$ & 4.96 & $2.972-08$ & 3.97 & $1.028-08$ & 4.12 \\
\hline
\end{tabular}

TABle 4. Problem with flow, $\nu=1$, nonconforming elements of order 4 .

\begin{tabular}{|c|c|c|c|c|c|c|c|c|c|c|c|c|}
\hline \multirow[b]{3}{*}{ Mesh } & \multicolumn{6}{|c|}{ Standard scheme } & \multicolumn{6}{|c|}{ Modified scheme } \\
\hline & \multicolumn{2}{|c|}{$\left\|\mathbf{u}-\mathbf{u}_{h}\right\|_{0}$} & \multicolumn{2}{|c|}{$\left|\mathbf{u}-\mathbf{u}_{h}\right|_{1, h}$} & \multicolumn{2}{|c|}{$\left\|p-p_{h}\right\|_{0}$} & \multicolumn{2}{|c|}{$\left\|\mathbf{u}-\mathbf{u}_{h}\right\|_{0}$} & \multicolumn{2}{|c|}{$\left|\mathbf{u}-\mathbf{u}_{h}\right|_{1, h}$} & \multicolumn{2}{|c|}{$\left\|p-p_{h}\right\|_{0}$} \\
\hline & error & ord & error & ord & error & ord & error & ord & error & ord & error & ord \\
\hline $2 \times 3$ & $3.171-05$ & & $8.345-04$ & & $5.138-04$ & & $4.606-05$ & & $1.154-03$ & & $1.170-03$ & \\
\hline $4 \times$ & $1.314-06$ & 4.59 & $7.562-05$ & 3.46 & $3.940-05$ & 3.70 & $1.928-06$ & 4.58 & $1.047-04$ & 3.46 & 7.654-05 & 3.93 \\
\hline $8 \times 12$ & $4.313-08$ & 4.93 & $5.211-06$ & 3.86 & $2.534-06$ & 3.96 & $7.003-08$ & 4.78 & $7.659-06$ & 3.77 & $4.326-06$ & 4.15 \\
\hline $16 \times 24$ & $1.345-09$ & 5.00 & $3.338-07$ & 3.96 & $1.550-07$ & 4.03 & 2.351-09 & 4.90 & $5.115-07$ & 3.90 & $2.442-07$ & 4.15 \\
\hline $32 \times 48$ & $4.163-11$ & 5.01 & $2.097-08$ & 3.99 & $9.446-09$ & 4.04 & $7.598-11$ & 4.95 & $3.293-08$ & 3.96 & $1.416-08$ & 4.11 \\
\hline
\end{tabular}

TABle 5. Problem with flow, $\nu=10^{-5}$, conforming elements of order 4 .

\begin{tabular}{|c|c|c|c|c|c|c|c|c|c|c|c|c|}
\hline \multirow[b]{3}{*}{ Mesh } & \multicolumn{6}{|c|}{ Standard scheme } & \multicolumn{6}{|c|}{ Modified scheme } \\
\hline & \multicolumn{2}{|c|}{$\left\|\mathbf{u}-\mathbf{u}_{h}\right\|_{0}$} & \multicolumn{2}{|c|}{$\left|\mathbf{u}-\mathbf{u}_{h}\right|_{1}$} & \multicolumn{2}{|c|}{$\left\|p-p_{h}\right\|_{0}$} & \multicolumn{2}{|c|}{$\left\|\mathbf{u}-\mathbf{u}_{h}\right\|_{0}$} & \multicolumn{2}{|c|}{$\left|\mathbf{u}-\mathbf{u}_{h}\right|_{1}$} & \multicolumn{2}{|c|}{$\left\|p-p_{h}\right\|_{0}$} \\
\hline & error & ord & error & ord & error & ord & error & ord & error & ord & error & ord \\
\hline $2 \times 3$ & $1.075+00$ & & $2.863+01$ & & 4.114-04 & & $4.613-05$ & & $1.217-03$ & & $3.744-04$ & \\
\hline $4 \times$ & $3.902-02$ & 4.78 & $2.033+00$ & 3.82 & $2.637-05$ & 3.96 & $2.017-06$ & 4.52 & $1.012-04$ & 3.59 & $2.486-05$ & 3.91 \\
\hline $8 \times 12$ & $1.308-03$ & 4.90 & $1.352-01$ & 3.91 & $1.632-06$ & 4.01 & $7.227-08$ & 4.80 & 7.074-06 & 3.84 & $1.575-06$ & 3.98 \\
\hline $16 \times 24$ & $4.224-05$ & 4.95 & 8.723-03 & 3.95 & $1.010-07$ & 4.01 & 2.401-09 & 4.91 & $4.646-07$ & 3.93 & $9.880-08$ & 4.00 \\
\hline $32 \times 48$ & $1.341-06$ & 4.98 & 5.539-04 & 3.98 & $6.279-09$ & 4.01 & $7.835-11$ & 4.94 & $2.972-08$ & 3.97 & 6.181-09 & 4.00 \\
\hline
\end{tabular}

\subsection{Problem with flow field}

We turn now to a problem with flow. The right-hand side of the Stokes's problem is chosen such that

$$
\mathbf{u}=\left(\begin{array}{c}
\partial_{y} \psi \\
-\partial_{x} \psi
\end{array}\right), \quad \psi=x^{2}(1-x)^{2} y^{2}(1-y)^{2}, \quad p=2 x^{2}(1-x) y(1-y)-\frac{1}{36}
$$

is the solution.

We will have first a look on the case $\nu=1$. The results for conforming and nonconforming discretisations of fourth order using the standard and the modified scheme are given in Tables 3 and 4 . We clearly see that the expected convergence orders of 4 for the (broken) $\mathrm{H}^{1}$-semi-norm of the velocity and the $\mathrm{L}^{2}$-norm of the pressure and of 5 for the $\mathrm{L}^{2}$-norm of the velocity are obtained in all cases. Moreover, the errors for the standard scheme are slightly smaller than the corresponding errors for the modified scheme.

The behaviour for the viscosity parameter $\nu=10^{-5}$ is completely different. The errors and convergence orders are given in Tables 5 and 6 . Although the standard scheme provides optimal convergence orders of 5 for the 
TABLE 6. Problem with flow, $\nu=10^{-5}$, nonconforming elements of order 4 .

\begin{tabular}{|c|c|c|c|c|c|c|c|c|c|c|c|c|}
\hline \multirow[b]{3}{*}{ mesh } & \multicolumn{6}{|c|}{ Standard scheme } & \multicolumn{6}{|c|}{ Modified scheme } \\
\hline & $\| \mathbf{u}-\mathbf{u}$ & $h \|_{0}$ & $\mid \mathbf{u}-\mathbf{u}_{h}$ & $1, h$ & $\| p-p_{l}$ & $\|_{0}$ & $\mid \mathbf{u}-\mathbf{u}$ & $h \|_{0}$ & $\mid \mathbf{u}-\mathbf{u}_{h}$ & $1, h$ & $\| p-p_{l}$ & ${ }_{2} \|_{0}$ \\
\hline & error & ord & error & ord & error & ord & error & ord & error & ord & error & ord \\
\hline $2 \times$ & $6.430-01$ & & $1.800+01$ & & $4.230-04$ & & $4.606-05$ & & $1.154-03$ & & $3.744-04$ & \\
\hline $4 \times$ & $2.756-02$ & 4.54 & $1.509+00$ & 3.58 & $2.708-05$ & 3.97 & $1.928-06$ & 4.58 & $1.047-04$ & 3.46 & $2.486-05$ & 3.91 \\
\hline $8 \times 12$ & $1.001-03$ & 4.78 & $1.073-01$ & 3.81 & $1.671-06$ & 4.02 & 7.003-08 & 4.78 & 7.659-06 & 3.77 & $1.575-06$ & 3.98 \\
\hline $16 \times 24$ & $3.361-05$ & 4.90 & $7.143-03$ & 3.91 & $1.032-07$ & 4.02 & 2.351-09 & 4.90 & $5.115-07$ & 3.90 & $9.880-08$ & 4.00 \\
\hline $32 \times 48$ & $1.088-06$ & 4.95 & $4.605-04$ & 3.96 & $6.406-09$ & 4.01 & $7.598-11$ & 4.95 & $3.293-08$ & 3.96 & 6.181-09 & 4.00 \\
\hline
\end{tabular}

TABle 7. Example with flow. Conforming discretisation of order $3,17 \times 23$-mesh.

\begin{tabular}{cccccc||c|c|c}
\hline & \multicolumn{4}{c}{ Standard scheme } & \multicolumn{3}{c}{ Modified scheme } \\
\cline { 2 - 7 }$\nu$ & $\left\|\mathbf{u}-\mathbf{u}_{h}\right\|_{0}$ & $\mathbf{u}-\left.\mathbf{u}_{h}\right|_{1}$ & $\left\|p-p_{h}\right\|_{0}$ & $\left\|\mathbf{u}-\mathbf{u}_{h}\right\|_{0}$ & $\mathbf{u}-\left.\mathbf{u}_{h}\right|_{1}$ & $\left\|p-p_{h}\right\|_{0}$ \\
\hline $10^{-0}$ & $2.0742 \mathrm{e}-08$ & $4.2338 \mathrm{e}-06$ & $4.4455 \mathrm{e}-06$ & $5.7559 \mathrm{e}-08$ & $1.1327 \mathrm{e}-05$ & $5.2358 \mathrm{e}-06$ \\
$10^{-1}$ & $1.7608 \mathrm{e}-07$ & $3.5676 \mathrm{e}-05$ & $4.4455 \mathrm{e}-06$ & $5.7559 \mathrm{e}-08$ & $1.1327 \mathrm{e}-05$ & $4.3587 \mathrm{e}-06$ \\
$10^{-2}$ & $1.7574 \mathrm{e}-06$ & $3.5603 \mathrm{e}-04$ & $4.4455 \mathrm{e}-06$ & $5.7559 \mathrm{e}-08$ & $1.1327 \mathrm{e}-05$ & $4.3491 \mathrm{e}-06$ \\
$10^{-3}$ & $1.7574 \mathrm{e}-05$ & $3.5602 \mathrm{e}-03$ & $4.4455 \mathrm{e}-06$ & $5.7559 \mathrm{e}-08$ & $1.1327 \mathrm{e}-05$ & $4.3490 \mathrm{e}-06$ \\
$10^{-4}$ & $1.7574 \mathrm{e}-04$ & $3.5602 \mathrm{e}-02$ & $4.4455 \mathrm{e}-06$ & $5.7559 \mathrm{e}-08$ & $1.1327 \mathrm{e}-05$ & $4.3490 \mathrm{e}-06$ \\
$10^{-5}$ & $1.7574 \mathrm{e}-03$ & $3.5602 \mathrm{e}-01$ & $4.4455 \mathrm{e}-06$ & $5.7559 \mathrm{e}-08$ & $1.1327 \mathrm{e}-05$ & $4.3490 \mathrm{e}-06$ \\
$10^{-6}$ & $1.7574 \mathrm{e}-02$ & $3.5602 \mathrm{e}+00$ & $4.4455 \mathrm{e}-06$ & $5.7559 \mathrm{e}-08$ & $1.1327 \mathrm{e}-05$ & $4.3490 \mathrm{e}-06$ \\
$10^{-7}$ & $1.7574 \mathrm{e}-01$ & $3.5602 \mathrm{e}+01$ & $4.4455 \mathrm{e}-06$ & $5.7559 \mathrm{e}-08$ & $1.1327 \mathrm{e}-05$ & $4.3490 \mathrm{e}-06$ \\
$10^{-8}$ & $1.7574 \mathrm{e}+00$ & $3.5602 \mathrm{e}+02$ & $4.4455 \mathrm{e}-06$ & $5.7566 \mathrm{e}-08$ & $1.1327 \mathrm{e}-05$ & $4.3490 \mathrm{e}-06$ \\
$10^{-9}$ & $1.7574 \mathrm{e}+01$ & $3.5602 \mathrm{e}+03$ & $4.4455 \mathrm{e}-06$ & $5.8112 \mathrm{e}-08$ & $1.1328 \mathrm{e}-05$ & $4.3490 \mathrm{e}-06$ \\
\hline
\end{tabular}

TABLE 8. Example with flow. Nonconforming discretisation of order $3,17 \times 23$-mesh.

\begin{tabular}{ccc|c|c||c|c|c}
\hline & \multicolumn{3}{c}{ Standard scheme } & \multicolumn{3}{c}{ Modified scheme } \\
\cline { 2 - 7 }$\nu$ & $\left\|\mathbf{u}-\mathbf{u}_{h}\right\|_{0}$ & $\mathbf{u}-\mathbf{u}_{h} \mid 1, h$ & $\left\|p-p_{h}\right\|_{0}$ & $\left\|\mathbf{u}-\mathbf{u}_{h}\right\|_{0}$ & $\mathbf{u}-\left.\mathbf{u}_{h}\right|_{1, h}$ & $\left\|p-p_{h}\right\|_{0}$ \\
\hline $10^{-0}$ & $7.2637 \mathrm{e}-08$ & $1.3500 \mathrm{e}-05$ & $4.4397 \mathrm{e}-06$ & $8.0962 \mathrm{e}-08$ & $1.5800 \mathrm{e}-05$ & $5.0577 \mathrm{e}-06$ \\
$10^{-1}$ & $1.5039 \mathrm{e}-07$ & $3.3942 \mathrm{e}-05$ & $4.4134 \mathrm{e}-06$ & $8.0962 \mathrm{e}-08$ & $1.5800 \mathrm{e}-05$ & $4.3566 \mathrm{e}-06$ \\
$10^{-2}$ & $1.3254 \mathrm{e}-06$ & $3.1327 \mathrm{e}-04$ & $4.4131 \mathrm{e}-06$ & $8.0962 \mathrm{e}-08$ & $1.5800 \mathrm{e}-05$ & $4.3490 \mathrm{e}-06$ \\
$10^{-3}$ & $1.3235 \mathrm{e}-05$ & $3.1300 \mathrm{e}-03$ & $4.4131 \mathrm{e}-06$ & $8.0962 \mathrm{e}-08$ & $1.5800 \mathrm{e}-05$ & $4.3490 \mathrm{e}-06$ \\
$10^{-4}$ & $1.3234 \mathrm{e}-04$ & $3.1299 \mathrm{e}-02$ & $4.4131 \mathrm{e}-06$ & $8.0962 \mathrm{e}-08$ & $1.5800 \mathrm{e}-05$ & $4.3490 \mathrm{e}-06$ \\
$10^{-5}$ & $1.3234 \mathrm{e}-03$ & $3.1299 \mathrm{e}-01$ & $4.4131 \mathrm{e}-06$ & $8.0962 \mathrm{e}-08$ & $1.5800 \mathrm{e}-05$ & $4.3490 \mathrm{e}-06$ \\
$10^{-6}$ & $1.3234 \mathrm{e}-02$ & $3.1299 \mathrm{e}+00$ & $4.4131 \mathrm{e}-06$ & $8.0962 \mathrm{e}-08$ & $1.5800 \mathrm{e}-05$ & $4.3490 \mathrm{e}-06$ \\
$10^{-7}$ & $1.3234 \mathrm{e}-01$ & $3.1299 \mathrm{e}+01$ & $4.4131 \mathrm{e}-06$ & $8.0962 \mathrm{e}-08$ & $1.5800 \mathrm{e}-05$ & $4.3490 \mathrm{e}-06$ \\
$10^{-8}$ & $1.3234 \mathrm{e}+00$ & $3.1299 \mathrm{e}+02$ & $4.4131 \mathrm{e}-06$ & $8.0962 \mathrm{e}-08$ & $1.5800 \mathrm{e}-05$ & $4.3490 \mathrm{e}-06$ \\
$10^{-9}$ & $1.3234 \mathrm{e}+01$ & $3.1299 \mathrm{e}+03$ & $4.4131 \mathrm{e}-06$ & $8.0967 \mathrm{e}-08$ & $1.5800 \mathrm{e}-05$ & $4.3490 \mathrm{e}-06$ \\
\hline
\end{tabular}

$\mathrm{L}^{2}$-norm of the velocity and of 4 for the (broken) $\mathrm{H}^{1}$-semi-norm of the velocity and the $\mathrm{L}^{2}$-norm of the pressure, the errors are much larger compared to the modified scheme which gives also the same optimal convergence orders. This observation holds for both conforming and nonconforming velocity discretisations. Furthermore, the pressure errors for both types of discretisations are identical, at least up to the leading four digits if the modified scheme is used. This interesting behaviour of the conforming and the nonconforming discrete pressures can be probably explained by (3.9), (3.6), and (3.7). Since both discrete pressure spaces are the same, the discrete pressure errors differ only up to velocity-dependent contributions which are proportional to $\nu$. Hence, these contributions are small for small viscosity parameters.

We will consider finally how the errors for conforming and nonconforming discretisations using the standard scheme or the modified scheme behave if the viscosity parameter $\nu$ changes. Tables 7 and 8 show the 
velocity errors in the $\mathrm{L}^{2}$-norm and the (broken) $\mathrm{H}^{1}$-semi-norm as well as the pressure error in the $\mathrm{L}^{2}$-norm. The calculations have used a fixed mesh of $17 \times 23$ axiparallel rectangles. We have chosen two prime numbers to minimise effects caused by symmetry. We clearly observe that velocity error increases like $\nu^{-1}$ for the both conforming and nonconforming discretisations if the standard scheme is applied. In contrast, the velocity errors for the modified scheme are independent of the parameter $\nu$. Furthermore, we see that the pressure error is almost constant, even for the standard scheme. This indicates that already the standard discretisation allows an accurate pressure approximation while the modification is needed to guarantee accurate velocity approximation in the case of small viscosity parameters.

\section{OutLOOK}

Future research will explore and evaluate the long-term potential of the proposed approach. Possible realworld applications of the approach exist even for the Stokes case and comprise coupled multi-physics flow problems. For the two following applications it is known that the exterior force may contain a dominating irrotational part in the sense of the Helmholtz decomposition:

- thermally-driven flows $[19,26]$ at high Rayleigh numbers; and

- the problem of the overlimiting current in microchannels [14] which is known from electrodialysis.

However, also u-dependent terms in the Navier-Stokes momentum balance may have large irrotational parts and would profit from reconstructing the velocity test functions. An example of such a flow can be found in [30]. In the master's thesis [28], it is further shown that in this benchmark replacing the standard discretization of the nonlinear convection term $\left(\left(\mathbf{u}_{h} \cdot \nabla\right) \mathbf{u}_{h}, \mathbf{v}_{h}\right)$ by $\left(\left(\mathbf{u}_{h} \cdot \nabla\right) \mathbf{u}_{h}, \Pi_{h} \mathbf{v}_{h}\right)$ leads to an accuracy improvement by about two refinement levels. However, such numerical results are only first promising indications which have to be investigated further and will be complemented by the investigation of fully nonlinear time-dependent flows.

\section{REFERENCES}

[1] T. Apel and G. Matthies, Nonconforming, anisotropic, rectangular finite elements of arbitrary order for the Stokes problem. SIAM J. Numer. Anal. 46 (2008) 1867-1891.

[2] D.N. Arnold and G. Awanou, Finite element differential forms on cubical meshes. Math. Comput. 83 (2014) 1551-1570.

[3] D. Boffi, F. Brezzi and M. Fortin, Mixed finite element methods and applications. Vol. 44 of Springer Ser. Comput. Math. Springer, Heidelberg (2013).

[4] M. Braack, E. Burman, V. John and G. Lube, Stabilized finite element methods for the generalized Oseen problem. Comput. Methods Appl. Mech. Engrg. 196 (2007) 853-866.

[5] J.H. Bramble and S.R. Hilbert, Estimation of linear functionals on Sobolev spaces with application to Fourier transforms and spline interpolation. SIAM J. Numer. Anal. 7 (1970) 112-124.

[6] C. Brennecke, A. Linke, C. Merdon and J. Schöberl, Optimal and pressure-independent $L^{2}$ velocity error estimates for a modified Crouzeix-Raviart Stokes element with BDM reconstructions. J. Comput. Math. 33 (2015) 191-208.

[7] S.C. Brenner and L.R. Scott, The mathematical theory of finite element methods. Vol. 15 of Texts Appl. Math. 3rd edition. Springer, New York (2008).

[8] F. Brezzi, J. Douglas, Jr. and LD. Marini, Recent results on mixed finite element methods for second order elliptic problems. In Vistas in Applied Mathematics, Transl. Ser. Math. Engrg. Optimization Software, New York (1986).

[9] F. Brezzi, J. Douglas, Jr., R. Durán and M. Fortin, Mixed finite elements for second order elliptic problems in three variables. Numer. Math. 51 (1987) 237-250.

[10] A. Buffa, C. de Falco and G. Sangalli, IsoGeometric Analysis: stable elements for the 2D Stokes equation. Int. J. Numer. Methods Fluids 65 (2011) 1407-1422.

[11] B. Cockburn, G. Kanschat and D. Schötzau, A note on discontinuous Galerkin divergence-free solutions of the Navier-Stokes equations. J. Sci. Comput. 31 (2007) 61-73.

[12] M. Crouzeix and P.-A. Raviart, Conforming and nonconforming finite element methods for solving the stationary Stokes equations. I. Rev. Française Automat. Informat. Recherche Opérationnelle Sér. Rouge 7 (1973) 33-75.

[13] O. Dorok, W. Grambow and L. Tobiska, Aspects of finite element discretizations for solving the Boussinesq approximation of the Navier-Stokes Equations. Notes on Numerical Fluid Mechanics: Numerical Methods for the Navier-Stokes Equations $\mathbf{4 7}$ (1994) 50-61.

[14] C. Druzgalski, M. Andersen and A. Mani, Direct numerical simulation of electroconvective instability and hydrodynamic chaos near an ion-selective surface. Phys. Fluids 25 (2013). 
[15] J.A. Evans and T.J.R. Hughes, Isogeometric divergence-conforming B-splines for the steady Navier-Stokes equations. Math. Models Methods Appl. Sci. 23 (2013) 1421-1478.

[16] R.S. Falk and M. Neilan, Stokes complexes and the construction of stable finite elements with pointwise mass conservation. SIAM J. Numer. Anal. 51 (2013) 1308-1326.

[17] M. Fortin, An analysis of the convergence of mixed finite element methods. RAIRO Anal. Numér. 11 (1977) $341-354$.

[18] L.P. Franca and T.J.R. Hughes, Two classes of mixed finite element methods. Comput. Methods Appl. Mech. Engrg. 69 (1988) $89-129$.

[19] K.J. Galvin, A. Linke, L.G. Rebholz and N.E. Wilson, Stabilizing poor mass conservation in incompressible flow problems with large irrotational forcing and application to thermal convection. Comput. Methods Appl. Mech. Engrg. 237-240 (2012) $166-176$.

[20] S. Ganesan, G. Matthies and L. Tobiska, On spurious velocities in incompressible flow problems with interfaces. Comput. Methods Appl. Mech. Engrg. 196 (2007) 1193-1202.

[21] J.-F. Gerbeau, C. Le Bris and M. Bercovier, Spurious velocities in the steady flow of an incompressible fluid subjected to external forces. Internat. J. Numer. Methods Fluids 25 (1997) 679-695.

[22] V. Girault and P.-A. Raviart, Finite element methods for Navier-Stokes equations. Theory and algorithms. Vol. 5 of Springer Ser. Comput. Math. Springer-Verlag, Berlin (1986).

[23] J. Guzmán and M. Neilan, Conforming and divergence-free Stokes elements in three dimensions. IMA J. Numer. Anal. 34 (2014) 1489-1508,.

[24] J. Guzmán and M. Neilan, Conforming and divergence-free Stokes elements on general triangular meshes. Math. Comput. 83 (2014) $15-36$.

[25] J.P. Hennart, J. Jaffré and J.E. Roberts, A constructive method for deriving finite elements of nodal type. Numer. Math. 53 (1988) 701-738.

[26] E.W. Jenkins, V. John, A. Linke and L.G. Rebholz, On the parameter choice in grad-div stabilization for the Stokes equations. Adv. Comput. Math. 40 (2014) 491-516.

[27] G. Kanschat and N. Sharma, Divergence-conforming discontinuous Galerkin methods and $C^{0}$ interior penalty methods. SIAM J. Numer. Anal. 52 (2014) 1822-1842.

[28] M. Koddenbrock, Effizienz und Genauigkeit einer divergenzfreien Diskretisierung für die stationären inkompressiblen NavierStokes-Gleichungen. Master's thesis, Freie Universität Berlin, Germany (2014).

[29] C. Lehrenfeld, Hybrid discontinuous Galerkin methods for incompressible flow problems. Master's thesis, RWTH Aachen, Germany (2010).

[30] A. Linke, Collision in a cross-shaped domain - a steady 2d Navier-Stokes example demonstrating the importance of mass conservation in CFD. Comput. Methods Appl. Mech. Engrg. 198 (2009) 3278-3286.

[31] A. Linke, On the role of the Helmholtz decomposition in mixed methods for incompressible flows and a new variational crime. Comput. Methods Appl. Mech. Engrg. 268 (2014) 782-800.

[32] G. Matthies, Inf-sup stable nonconforming finite elements of higher order on quadrilaterals and hexahedra. ESAIM: M2AN 41 (2007) 855-874.

[33] G. Matthies and L. Tobiska, The inf-sup condition for the mapped $Q_{k}-P_{k-1}^{\text {disc }}$ element in arbitrary space dimensions. Computing 69 (2002) 119-139.

[34] G. Matthies and L. Tobiska, Inf-sup stable non-conforming finite elements of arbitrary order on triangles. Numer. Math. 102 (2005) 293-309.

[35] G. Matthies and L. Tobiska, Mass conservation of finite element methods for coupled flow-transport problems. Int. J. Comput. Sci. Math. 1 (2007) 293-307.

[36] J.-C. Nédélec, Mixed finite elements in $\mathbb{R}^{3}$. Numer. Math. 35 (1980) 315-341.

[37] M.A. Olshanskii, G. Lube, T. Heister and J. Löwe, Grad-div stabilization and subgrid pressure models for the incompressible Navier-Stokes equations. Comput. Methods Appl. Mech. Engrg. 198 (2009) 3975-3988.

[38] M.A. Olshanskii and A. Reusken, Grad-div stabilization for Stokes equations. Math. Comput. 73 (2004) $1699-1718$.

[39] P.-A. Raviart and J.M. Thomas, A mixed finite element method for 2nd order elliptic problems. In Mathematical aspects of finite element methods. Proc. Conf., Consiglio Naz. delle Ricerche, C.N.R., Rome, 1975. Vol. 606 of Lect. Notes Math. Springer, Berlin (1977) 292-315.

[40] H.-G. Roos, M. Stynes and L. Tobiska, Robust numerical methods for singularly perturbed differential equations. Convectiondiffusion-reaction and flow problems. Vol. 24 of Springer Ser. Comput. Math. 2nd edition. Springer-Verlag, Berlin (2008).

[41] L.R. Scott and M. Vogelius, Norm estimates for a maximal right inverse of the divergence operator in spaces of piecewise polynomials. RAIRO Modél. Math. Anal. Numér. 19 (1985) 111-143.

[42] J. Wang, Y. Wang and X. Ye, A posteriori error estimation for an interior penalty type method employing $H$ (div) elements for the Stokes equations. SIAM J. Sci. Comput. 33 (2011) 131-152.

[43] S. Zhang, A new family of stable mixed finite elements for the 3D Stokes equations. Math. Comput. 74 (2005) 543-554. 